\title{
Developmental SHP2 dysfunction underlies cardiac hypertrophy in Noonan syndrome with multiple lentigines
}

\author{
Jessica Lauriol, ${ }^{1}$ Janel R. Cabrera, ${ }^{1}$ Ashbeel Roy, ${ }^{1}$ Kimberly Keith, ${ }^{1}$ Sara M. Hough, ${ }^{1}$ Federico Damilano, ${ }^{1}$ Bonnie Wang, ${ }^{1}$ \\ Gabriel C. Segarra, ${ }^{2}$ Meaghan E. Flessa, ${ }^{2}$ Lauren E. Miller, ${ }^{2}$ Saumya Das, ${ }^{1}$ Roderick Bronson, ${ }^{3}$ Kyu-Ho Lee, ${ }^{2}$ and Maria I. Kontaridis ${ }^{1,4}$ \\ 'Department of Medicine, Division of Cardiology, Beth Israel Deaconess Medical Center, Boston, Massachusetts, USA. ²Department of Pediatrics and Department of Obstetrics and Gynecology, \\ Medical University of South Carolina, Charleston, South Carolina, USA. ${ }^{3}$ Department of Pathology and ${ }^{4}$ Department of Biological Chemistry and Molecular Pharmacology, \\ Harvard Medical School, Boston, Massachusetts, USA.
}

\begin{abstract}
Hypertrophic cardiomyopathy is a common cause of mortality in congenital heart disease (CHD). Many gene abnormalities are associated with cardiac hypertrophy, but their function in cardiac development is not well understood. Loss-of-function mutations in PTPN11, which encodes the protein tyrosine phosphatase (PTP) SHP2, are implicated in CHD and cause Noonan syndrome with multiple lentigines (NSML), a condition that often presents with cardiac hypertrophic defects. Here, we found that NSML-associated hypertrophy stems from aberrant signaling mechanisms originating in developing endocardium. Trabeculation and valvular hyperplasia were diminished in hearts of embryonic mice expressing a human NSML-associated variant of SHP2, and these defects were recapitulated in mice expressing NSML-associated SHP2 specifically in endothelial, but not myocardial or neural crest, cells. In contrast, mice with myocardial- but not endothelial-specific NSML SHP2 expression developed ventricular septal defects, suggesting that NSML-associated mutations have both cell-autonomous and nonautonomous functions in cardiac development. However, only endothelial-specific expression of NSML-associated SHP2 induced adult-onset cardiac hypertrophy. Further, embryos expressing the NSML-associated SHP2 mutation exhibited aberrant AKT activity and decreased downstream forkhead box P1 (FOXP1)/FGF and NOTCH1/EPHB2 signaling, indicating that SHP2 is required for regulating reciprocal crosstalk between developing endocardium and myocardium. Together, our data provide functional and disease-based evidence that aberrant SHP2 signaling during cardiac development leads to CHD and adult-onset heart hypertrophy.
\end{abstract}

\section{Introduction}

With a prevalence of approximately 1:100 children and over 500,000 adult cases reported in the United States alone, congenital heart disease (CHD) remains the most common birth defect worldwide and the major cause of birth defect-related deaths (1). Hypertrophic cardiomyopathy (HCM), which affects the cardiac muscle wall, is a common cause of mortality and morbidity in CHD patients (2). Although initially a compensatory response to increased cardiac stress, over time, HCM leads to dilation and heart failure. Gene abnormalities that affect hypertrophic signaling molecules have been identified, but their mechanistic roles in the developing heart that lead to this phenotype remain poorly understood.

Cardiac development consists of a concordant and sequential series of morphogenic events. Briefly, following formation of the primitive heart tube, the heart undergoes elongation and looping and initiates a series of proliferation, migration, and differentiation events that lead to the formation of a fully functional heart containing 4 chambers separated by valves and septa and connected to the extracardiac circulation via inflow and outflow

Conflict of interest: The authors have declared that no conflict of interest exists. Submitted: December 8, 2014; Accepted: May 9, 2016.

Reference information: J Clin Invest. 2016;126(8):2989-3005. doi:10.1172/JCI80396. tracts (OFT) (3). Importantly, these developmental events are controlled by intercellular communications mediated by multiple cardiac cell lineages, including endothelium, myocardium, and neural crest. Specifically, cell-cell communication occurs between cardiac mesoderm progenitors, neural crest-derived cells, and pharyngeal endoderm to regulate heart tube elongation, looping, and morphogenesis of the OFT (4). In addition, crosstalk between endocardium and myocardium occurs to control both the formation of cardiac valves (5) and ventricular cardiomyocyte differentiation and proliferation (6).

Multiple signaling pathways are involved in regulating these complex developmental events, the perturbation of which can cause various cardiac defects, including abnormal valves, membranous septa, and/or myocardial defects. Two major players directly involved in cardiac morphogenic events and the reciprocal crosstalk therein are NOTCH1 and forkhead box P1 (FOXP1). $\mathrm{NOTCH}$ signaling from mesoderm progenitors is required to mediate interactions with migrating cardiac neural crest cells, orchestrating appropriate OFT development (7). In addition, during early valve development, NOTCH1 signaling in endocardial cells induces BMP2/10 expression in the adjacent myocardium, initiating endocardial-to-mesenchymal transition (EndoMT) (8). Concomitantly, during ventricular chamber formation, both 
FOXP1 and NOTCH1 expression in the endocardium lead to paracrine production of FGFs, BMPs, and neuregulin to induce trabeculation, myocardial proliferation, and differentiation $(7,9)$. Importantly, aberrant regulation of these critical pathways leads to developmental cardiac defects. For example, NOTCH pathway mutations have been causally linked to dysmorphic pulmonic valve, hypertrophy, and ventricular septal defect (VSD) $(10,11)$ Similarly, Foxp1 $1^{-/-}$embryos develop severe defects in OFT and cardiac cushion formation, have thin ventricular myocardial walls, and lack proper ventricular septation, leading to embryonic death at E14.5 (12). Whether aberrant regulation of these pathways is involved in mediating developmental signaling events that induce myocardial hypertrophy, however, remains unknown.

One nonsarcomeric signaling protein whose aberrant regulation is involved in CHD-associated cardiac hypertrophy is the ubiquitously expressed $\mathrm{SH} 2$ domain-containing protein tyrosine phosphatase (PTP) SHP2, encoded by the PTPN11 gene. Germline mutations in PTPN11 cause more than $45 \%$ of incidences of Noonan syndrome (NS) (OMIM 163950) (13) and virtually all NS with multiple lentigines (NSML) (called LEOPARD syndrome; OMIM 151100) (14), both of which belong to a group of collective disorders called RASopathies. Despite the fact that NS and NSML are allelic variant disorders that present with similar phenotypes, including cutaneous-cardio-craniofacial defects and retardation of growth, they display distinct spectra of cardiac involvement. NSML principally presents with HCM, the major morbidity-associated feature of the disorder $(15,16)$, but patients can also have valve defects, electrocardiographic conduction abnormalities, and rarely, pulmonic stenosis (14). In contrast, NS-causing PTPN11 mutations are rarely associated with $\mathrm{HCM}$, and patients present more frequently with pulmonic stenosis and valvuloseptal defects (17).

Crystal structure analysis and computational modeling have provided mechanistic insights regarding the consequences of SHP2 mutations $(18,19)$. Normally, SHP2 phosphatase activity is regulated by an intramolecular conformational switch that oscillates between a closed and open conformation upon binding to phosphotyrosyl-containing proteins. Both NSML and NS mutations facilitate the open conformation and lead to enhanced interaction of SHP2 with binding partners, including cell membrane receptors and scaffolding adapters (20). However, whereas NS mutations are gain of function (GOF) and potentiate SHP2 phosphatase activity (21-26), all NSML mutations identified so far affect conserved residues important for PTP catalysis and are loss of function (LOF) for the phosphatase activity (20, 27-30).

SHP2 plays a key role in RAS/ERK activation in most, if not all, tyrosine kinase, cytokine, G protein-coupled receptors, and integrin-mediated signaling pathways (31). However, SHP2 is also involved in PI3K/AKT signaling, although its role depends on the type of receptor tyrosine kinase implicated. For example, in PDGFR and IGF receptor (IGFR) signaling, SHP2 is required for PI3K activation, likely through an adaptor-like activity of its SH2 domains (32). In contrast, in EGF receptor (EGFR) signaling, SHP2 negatively regulates PI3K activity by dephosphorylating PI3K-binding sites on Grb2-associated binding protein 1 (GAB1) (33) and insulin receptor substrate (IRS1) (34).

Dysregulation of the PI3K/AKT pathway has been causally linked to the cardiac pathophysiology of NSML. In vitro,
$S H P 2^{\mathrm{Y} 279 C}$ and $S H P 2^{\text {T468M }}$ mutants promote a hypertrophic phenotype in cardiomyocytes through impaired GAB1 dephosphorylation, leading to increased PI3K/AKT activity (35). Furthermore, we showed previously that hearts from an NSML mouse model using a knockin of the Y279C Ptpn11 allele (Shp2 $2^{\mathrm{Y} 279 C /+}$ mice, also called $P t p n 11^{\mathrm{Y} 279 \mathrm{C} / \mathrm{+}}$ mice) have elevated PI3K/mTOR activity and that rapamycin-mediated mTOR inhibition both prevents and reverses adult-onset HCM (28). Transgenic overexpression of the NSML mutant SHP2 ${ }^{\mathrm{Q} 510 E}$ specifically in cardiomyocytes confirms that AKT/mTOR activity is a driver of NSML-associated HCM (29). However, in that study, HCM was observed only when the transgene was expressed under the control of a developmental cardiomyocyte-specific promoter $M y h 7$ ( $\beta$-myosin heavy chain [ $\beta$-MHC]); no phenotype was observed with the $\alpha$-MHC promoter, which is expressed postnatally in the ventricles, suggesting that adult-onset NSML-associated HCM is triggered by developmental events affecting cardiomyocytes. Whether other cardiac cell lineages are involved in NSML-associated developmental defects and/or HCM remains unknown.

In this regard, upregulation of the RAS/ERK pathway in the endocardium was previously shown to be responsible for most of the cardiac defects in NS; however, since NS-causing PTPN11 mutations are not associated with HCM (17) and the biochemical properties of NS- and NSML-associated SHP2 mutations are opposing, it is likely that the mechanistic role for NSML in cardiac development is distinct. To determine whether NSML-associated HCM stems from aberrant cardiac developmental events, we utilized the $S h p 2^{\mathrm{Y} 279 \mathrm{C} /{ }^{+}}$mouse model. Our findings are the first, to our knowledge, to indicate that SHP2 NSML-causing mutations have both cell-autonomous and nonautonomous effects on the heart, leading to developmental defects and adult-onset HCM. Mechanistically, we found that an increased activation of AKT in NSML embryos and the associated decreases in downstream FOXP1/ FGF and NOTCH1/EPHB2 signaling mediate these effects by perturbing the pathways necessary for reciprocal crosstalk between developing endocardium and myocardium.

\section{Results}

Homozygous NSML mice are viable and develop early onset cardiac hypertrophy. Shp2 deletion results in completely penetrant embryonic lethality during midgestation, with defects in patterning observed at gastrulation (36); therefore, Ptpn11 phosphatase activity was thought to be required for survival. However, despite having only minimal levels of SHP2 phosphatase activity (Figure 1A), mice born with homozygous expression of NSML (Shp2 $2^{\mathrm{Y} 279 C / \mathrm{Y} 279 \mathrm{C})}$ were viable, albeit at reduced Mendelian ratios (Supplemental Table 1). These data provocatively suggest that non-phosphatase-mediated functions of SHP2 are critical regulators of early embryonic development. Phenotypically, adult $S h p 2^{\mathrm{Y} 279 \mathrm{C} /{ }^{+}}$and $\operatorname{Sh} p 2^{\mathrm{Y} 279 \mathrm{C} / \mathrm{Y} 279 \mathrm{C}}$ mice showed gene dosage-dependent inhibition of growth and exaggerated craniofacial abnormalities (Figure 1B) as compared with WT $S h p 2^{+/+}$mice. Interestingly, bone structure to body length ratios were disproportionate in NSML mice, as tibia lengths in $S h p 2^{\mathrm{Y} 279 \mathrm{C} /+}$

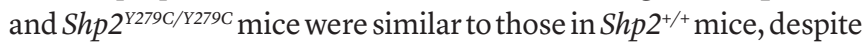
gene dosage-dependent decreases in both body lengths and body weights (ref. 28, Figure 1B, and Supplemental Figure 1A; supplemental material available online with this article; doi:10.1172/ 
A

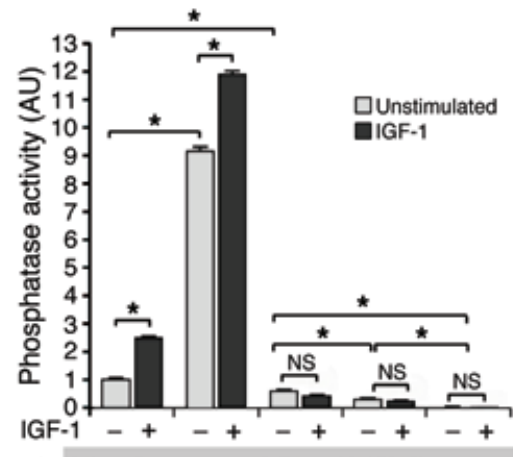

Ig L-chain

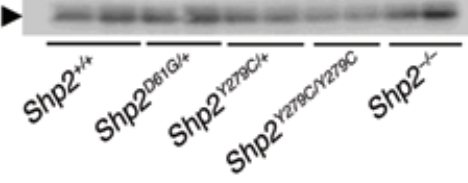

B
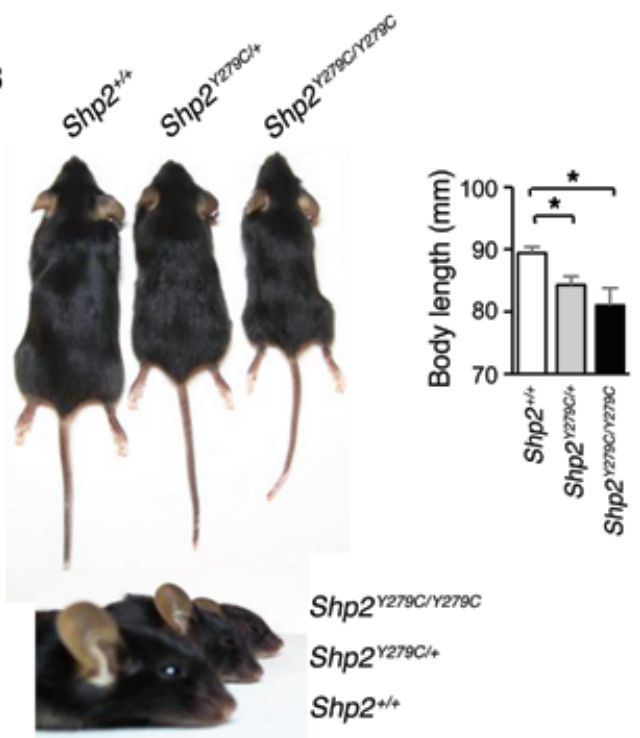

Shp2 $2^{\text {r279c/r279C }}$

Shp2 $2^{\text {rezoc/* }}$

Shp2 $2^{*+*}$
C
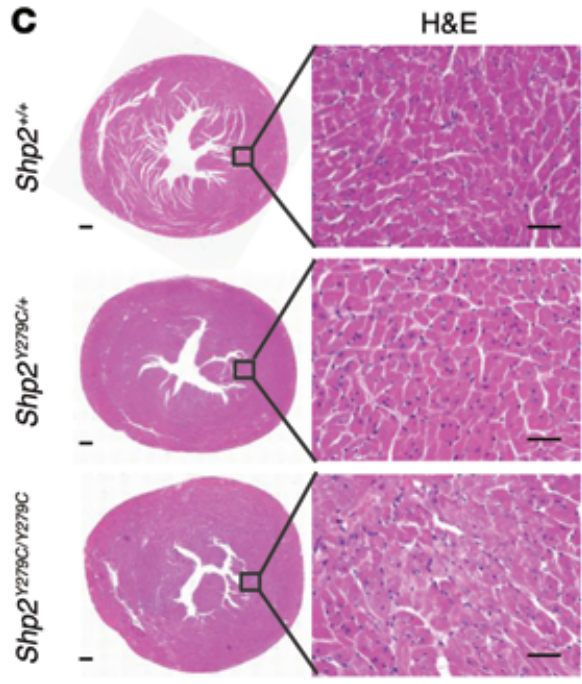

Reticulin
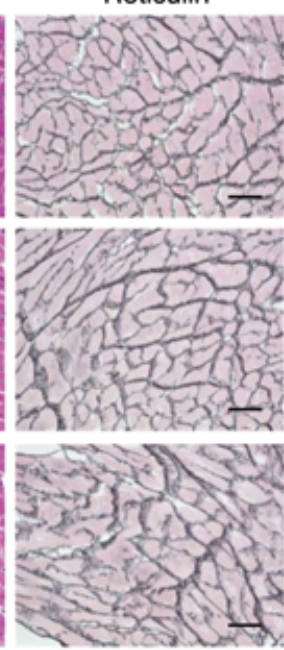

Trichrome
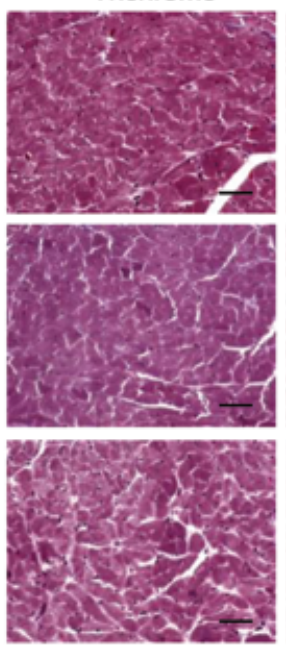
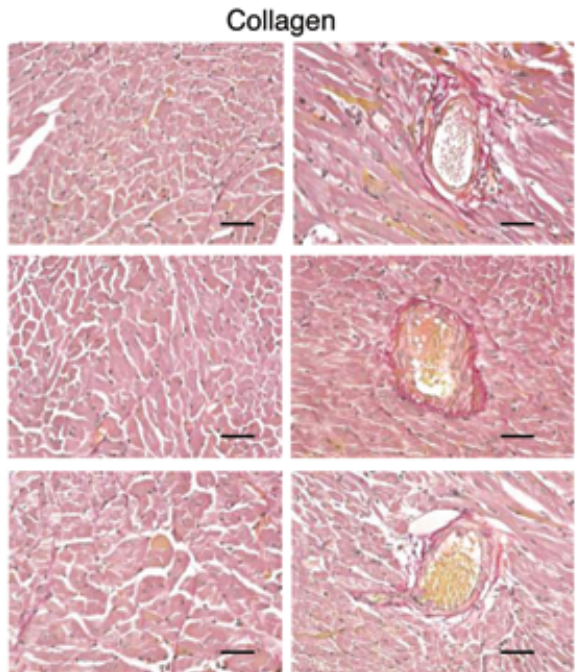

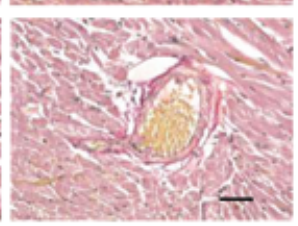

D

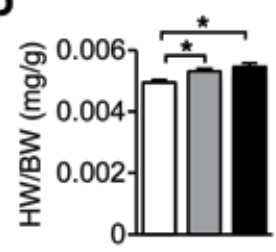

G

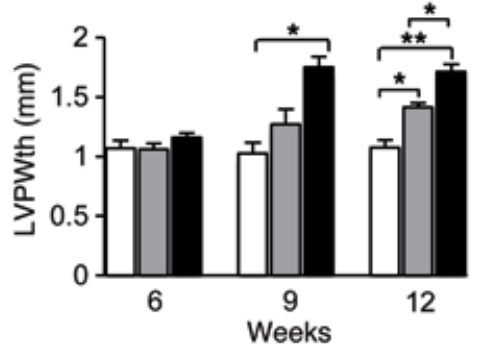

$\mathbf{E}$

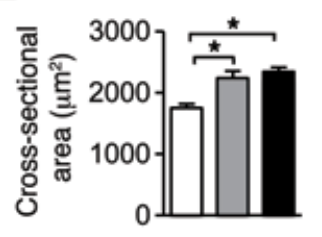

$\mathbf{F}$

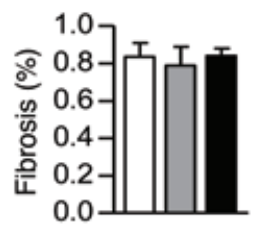

H

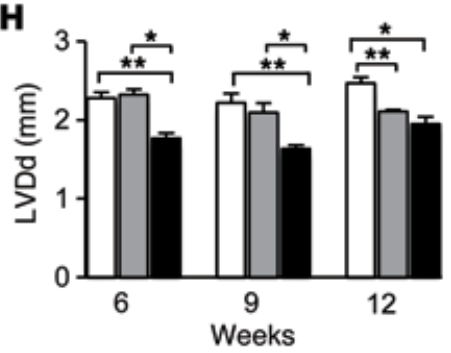

Shp2*/*

$\square$ Shp2 $2^{\text {Y279C/* }}$

Shp $2^{\text {Y2796/r279C }}$

Figure 1. The NSML phenotype is gene dosage dependent. (A) Primary MEFs were isolated from control

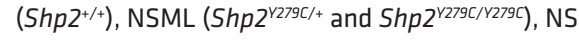

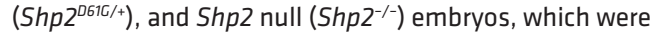
either left unstimulated or were stimulated with IGF-1 (10 nM) for 10 minutes, and SHP2 immune complex PTP assays were conducted using pNPP as substrate. Immunoblot control of immunoprecipitated SHP2 showing comparable recovery is shown. (B) Representative photographs of littermate mice at 12 weeks, showing relative lengths and craniofacial abnormalities of NSML mice. (C) Representative transverse cross-sections of whole hearts and H\&E-, reticulin-, Masson-trichrome-, and collagenstained sections. Scale bars: $200 \mu \mathrm{m}$ (far left column); 50 $\mu \mathrm{m}$ (all other columns). (D) Heart-to-body weight (HW/ BW) ratios. (E) Cardiomyocyte cross-sectional areas, $n=100-300$ cells/genotype. (F) Percentage of fibrosis in

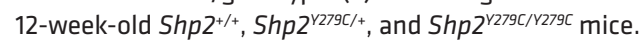
$n=3-7$ mice/group. Echocardiographic analyses of (G) LVPWth and (H) LVDd in 6-, 9-, and 12-week-old Shp2 ${ }^{+/+}$,

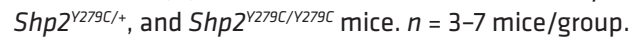
Data represent mean $\pm \mathrm{SEM}$. ${ }^{*} P<0.05 ;{ }^{* *} P<0.01$. $P$ values were derived from 1-way ANOVA with Bonferroni's post-test when ANOVA was significant. 
A

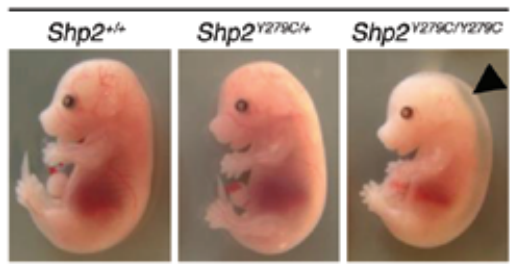

B

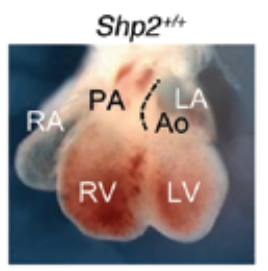

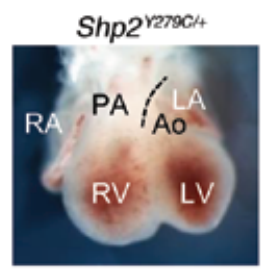

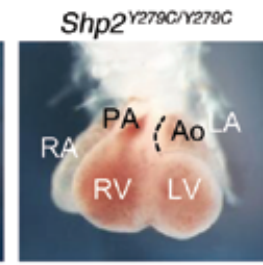

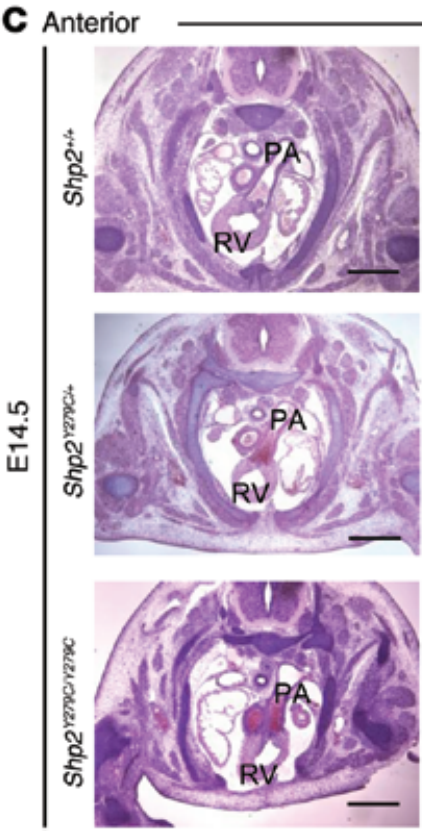
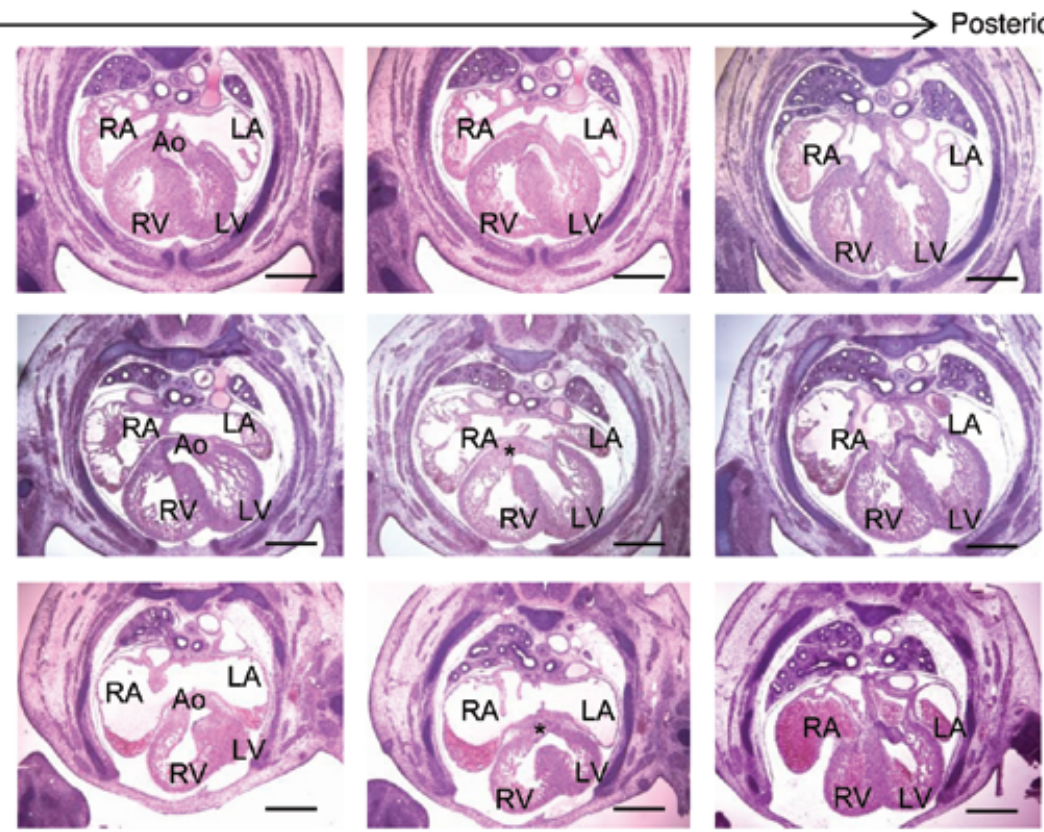

Figure 2. NSML embryo hearts at E14.5 display alignment and VSDs, leading to partial embryonic lethality. (A) Representative photomicrographs of E15.5 littermate embryos from heterozygous Shp2 $2^{1279 c /+}$ mating. (B) Bright-field whole-mount frontal view of hearts from E14.5 littermate embryos. Dotted lines delineate the leftmost border of the pulmonary artery, highlighting the relative leftward shift of the pulmonary artery and corresponding rightward shift of aorta in Shp2 $2^{1279 c / 1279 C}$ embryo hearts. (C) Cardiac morphology of anterior to posterior H\&E-stained heart sections of littermate embryos. Note, both

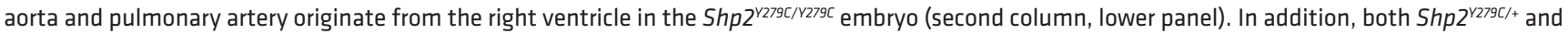

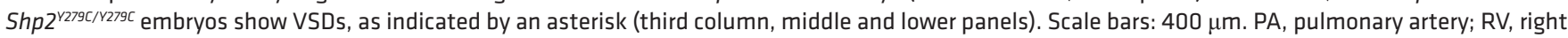
ventricle; LV, left ventricle; Ao, aorta; LA, left atria; RA, right atria.

JCI80396DS1). In line with the NSML phenotype, 12-week-old $\operatorname{Sh} p 2^{\mathrm{Y} 279 \mathrm{C} /+}$ and $\operatorname{Sh} p 2^{\mathrm{Y} 279 \mathrm{C} / \mathrm{Y} 27 \mathrm{CC}}$ mice also had thickened hearts with enlarged nuclei and increased interstitial spacing, increased heartto-body weight ratios, and enlarged cardiomyocyte areas, as compared with $S h p 2^{+/+}$mice (Figure 1, C-E). No changes in lung weight (Supplemental Figure 1B) or in fibrosis or collagen deposition were noted at this age in NSML mice (Figure 1, C and F). Consistent with the enlarged heart phenotype, individual cardiomyocytes isolated from $S h p 2^{\mathrm{Y} 279 \mathrm{C} /+}$ and $S h p 2^{\mathrm{Y} 279 \mathrm{C} / \mathrm{Y279C}}$ mice were also larger, with significantly increased widths and lengths, as compared with $S h p 2^{+/+}$ cells (Supplemental Figure 2, A and B). Moreover, we observed mRNA expression level shifts from the adult $\alpha$-MHC isoform to the fetal $\beta$-MHC isoform in NSML hearts, demonstrating an induction of the fetal gene program in response to pathological hypertrophy in these mice (Supplemental Figure 2C). Functionally, echocardiography revealed a gene dosage-dependent hypertrophic phenotype in NSML mice, with reduced left ventricular chamber dimension (LVDd) and increased left ventricular posterior wall thickness (LVPWth) at 12 weeks in $S h p 2^{\mathrm{Y} 279 \mathrm{C} /+}$ mice and as early as 6 weeks

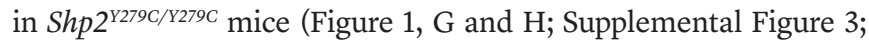
and Supplemental Table 2). Indeed, while we noted a significant number of deaths $(\sim 30 \%)$ in the $S h p 2^{\mathrm{Y} 279 \mathrm{C} / \mathrm{+}}$ mice by 52 weeks (28), we observed an accelerated death rate in homozygous mice, with approximately $50 \%$ of the $S h p 2^{\mathrm{Y279C/Y279C}}$ mice dying between 8 and 12 months of age (data not shown). Together, these data suggest that the severity of the NSML phenotype is gene dosage dependent and that the phosphatase function of SHP2 may be dispensable during early embryonic development.

Homozygous expression of NSML leads to partial embryonic lethality and multiple cardiac defects involving endocardium and myocardium. To further investigate the reduced Mendelian ratio of $S h p 2^{\text {Y2279C/Y279C }}$ mice, we conducted a retrograde timed mating analysis, which revealed that an embryonic lethality event occurs between E14.5 and E15.5 (Supplemental Table 1). At this stage,

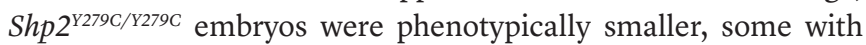
significant hydrops (Figure 2A). To determine whether the lethality in NSML mice might stem from abnormal placental development, we isolated and analyzed the placentae from developing 
A

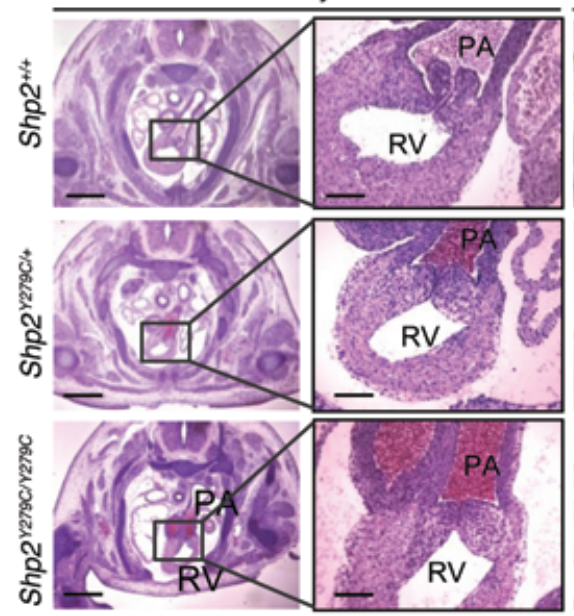

D
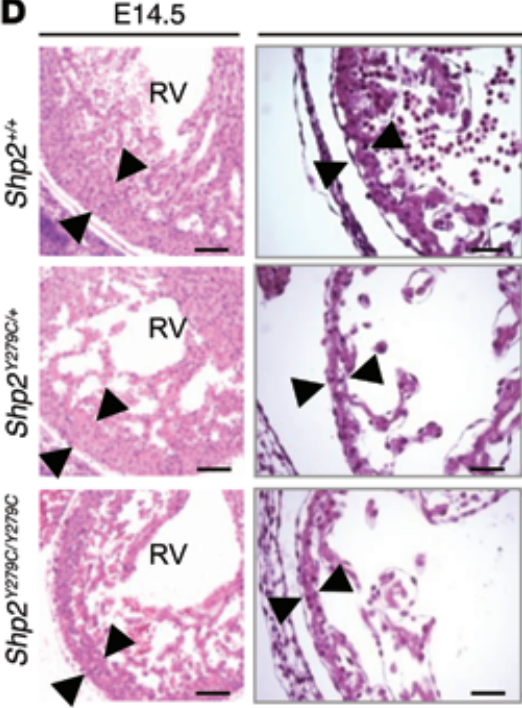

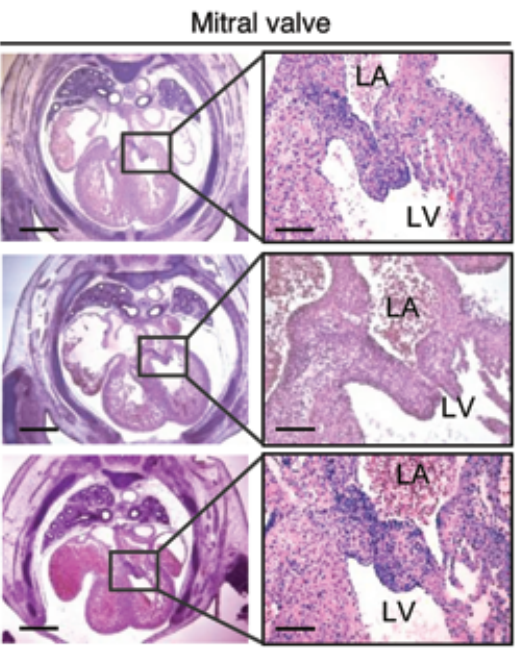

B

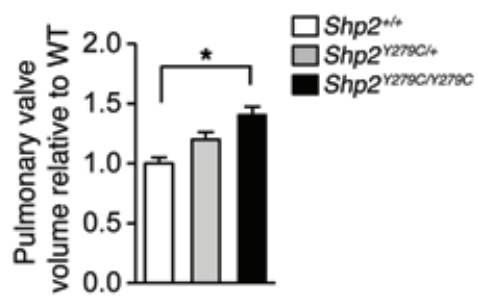

C

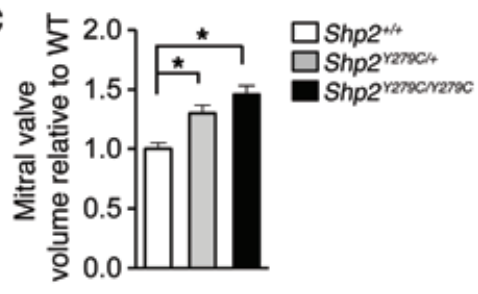

E

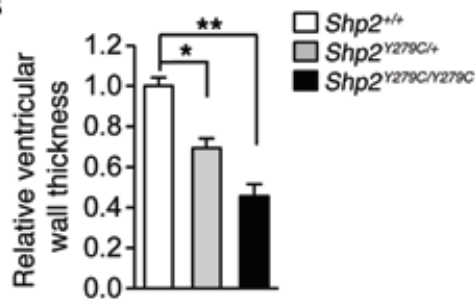

$\mathbf{F}$

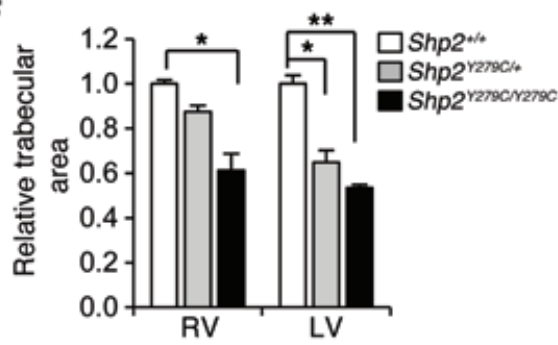

Figure 3. NSML embryo hearts display enlarged endocardial cushions, decreased trabeculation, and a thinner myocardial wall. (A) H\&E staining of endocardial cushions of developing pulmonary and mitral valve leaflets at E14.5. Scale bars: $400 \mu \mathrm{m} ; 100 \mu \mathrm{m}$ (inset). Quantification of (B) pulmonary and (C) mitral valve volumes of NSML hearts relative to control $\left(S h p 2^{+/+}\right) . n=3$ embryos/group. (D) H\&E staining of ventricular wall at E14.5 and E10.5, respectively. Note the thinning of the compact zone (black arrowheads) and a decreased trabecular area in NSML embryos. Scale bars: $200 \mu \mathrm{m} ; 100 \mu \mathrm{m}$ (insets). (E) Quantification of ventricular wall thickness at E14.5. $n=3-4$ embryos, with at least 10 sections per embryo. (F) Quantification of cardiac trabecular area at E10.5, normalized against ventricular volume and expressed as relative to control $\left(S h p 2^{+/+}\right) . n=3-4$ embryos per genotype. Data represent mean \pm SEM. ${ }^{*} P<0.05 ;{ }^{* *} P<0.01$. $P$ values were derived from 1-way ANOVA with Bonferroni's post-test when ANOVA was significant.

$\operatorname{Sh} p 2^{+/+}, \operatorname{Sh} p 2^{\mathrm{Y} 279 \mathrm{C} /+}$, and $\operatorname{Sh} p 2^{\mathrm{Y} 279 \mathrm{C} / \mathrm{Y} 279 \mathrm{C}}$ embryos. We observed no gross placental or umbilical vascular anomalies and found a normal histological appearance of the villous trophoblast (VT) and trophoblast giant cell (TGC) layers of the placenta. There was no evidence of decidual abnormalities at the maternal interface, no hemorrhaging, and no abruption in any of the mouse genotypes, suggesting that the lethality in $S h p 2^{Y 279 C / Y 279 C}$ mice was unlikely to be a consequence of abnormal placental development (Supplemental Figure 4). Therefore, we next analyzed NSML embryos to determine the effects of the mutation on heart development. Shp $2^{\mathrm{Y} 279 \mathrm{C} /+}$ and $S h p 2^{\mathrm{Y} 279 \mathrm{C} / \mathrm{Y} 279 \mathrm{C}}$ embryos displayed initial evidence of cardiac looping and septal alignment defects at E14.5, with the appearance of abnormal curvature and aberrant relative positioning of the developing aorta and pulmonary artery (Figure 2B).
Indeed, and in approximately $10 \%$ of $\operatorname{Shp} 2^{\mathrm{Y} 279 \mathrm{C} / \mathrm{Y} 279 \mathrm{C}}$ embryos, these defects appeared to be severe enough to result in relative dextrapositioning of the aorta (Figure 2C). In addition, both $S h p 2^{\mathrm{Y} 279 \mathrm{C} /+}$ and $\operatorname{Shp} 2^{\mathrm{Y} 279 \mathrm{C} / \mathrm{Y} 279 \mathrm{C}}$ hearts showed VSD, as indicated by a lack of fusion between the ventricular septum and the atrio-ventricular cushions (AVC) (Figure 2C). Interestingly, while the VSD persisted in homozygous embryos, it resolved by E15.5 in heterozygous mice (data not shown), suggesting that this phenotype may be caused by developmental delay due to the LOF activity of SHP2 in NSML.

We also observed that $S h p 2^{\mathrm{Y} 279 \mathrm{C} /+}$ and $S h p 2^{\mathrm{Y} 279 \mathrm{C} / \mathrm{Y} 279 \mathrm{C}}$ embryos had dosage-dependent enlarged and amorphic pulmonary and mitral valve leaflets, with significantly increased valve volumes, as compared with $S h p 2^{+/+}$embryos (Figure 3, A-C). In addition, we noted defective cardiac chamber maturation in E14.5 Shp $2^{\text {Y279C/+ }}$ 

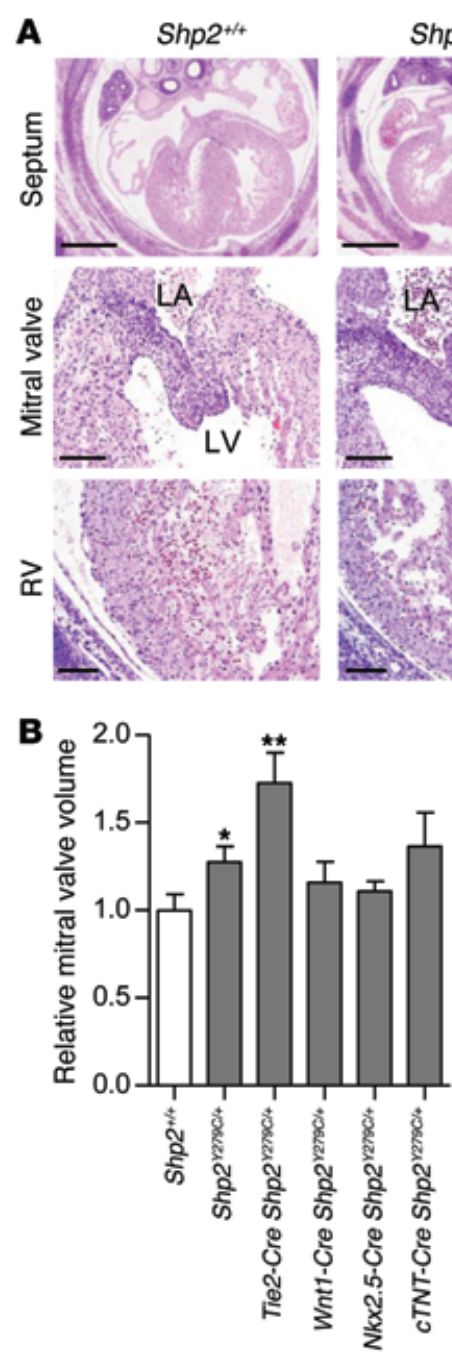

Shp2 $2^{\text {r279C/4 }}$
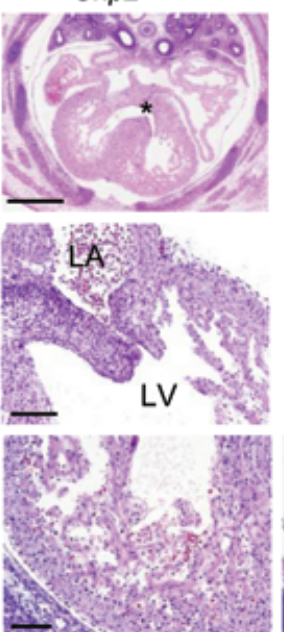

Tie2-Cre Shp2 $2^{\text {Y2796/4 }}$
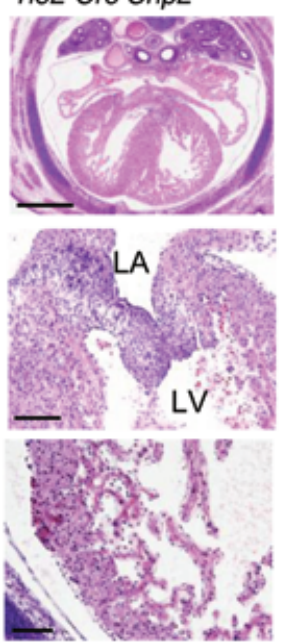

Wnt1-Cre Shp2 $2^{\text {r279c/ }+}$
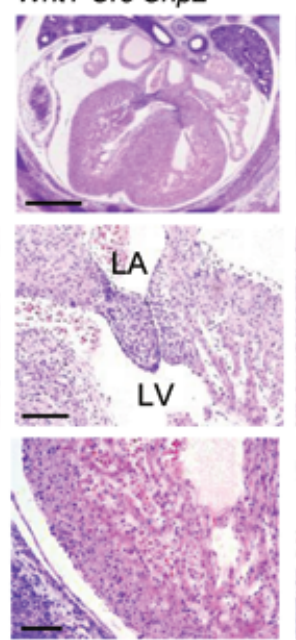
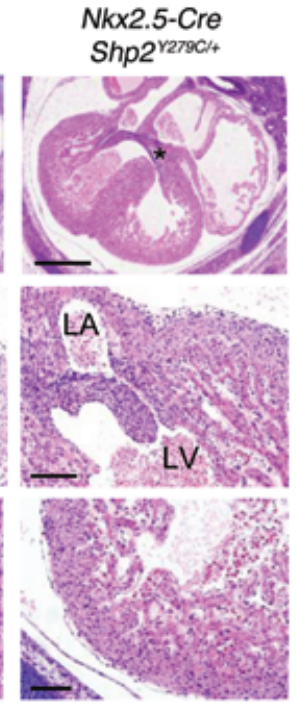

cTNT-Cre Shp2 $2^{\text {r279C/4 }}$

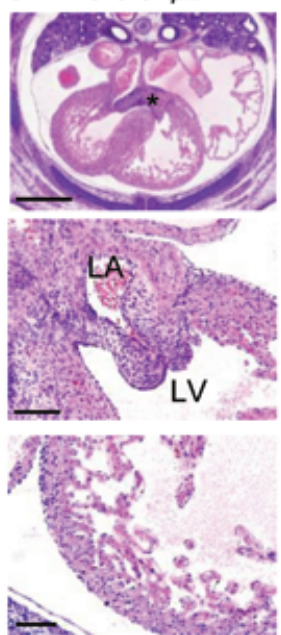

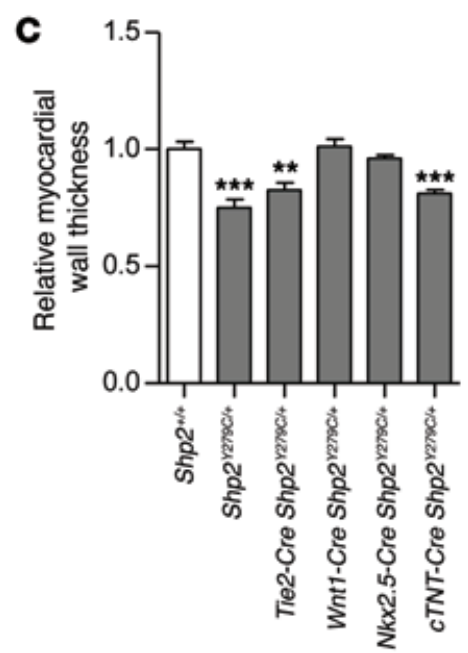

Figure 4. Endocardial- and myocardial-specific expression of NSML mutations both contribute to the embryonic cardiac defects observed in ubiquitously expressing NSML mice. (A) H\&E-stained heart sections of ubiquitous and lineage-specific expressing NSML embryos at E14.5. Scale bars: $400 \mu \mathrm{m}$ (top row); $100 \mu \mathrm{m}$ (second and third rows). Quantification of (B) mitral valve volume and (C) myocardial wall thickness relative to control $\left(\mathrm{Shp2}^{+/+}\right)$. $n=3-5$ embryos per group. Data represent mean \pm SEM. ${ }^{*} P<0.05 ;{ }^{*} P<0.01 ;{ }^{* *} P<0.005$. $P$ values were derived from 2-tailed Student's $t$ test.

and $S h p 2^{Y 279 C / / 279 C}$ embryos (Figure 3D) and a significant decrease in ventricular wall thickness (Figure 3E), suggesting a preclinical abnormality associated with HCM and a possible cause of cardiac failure and intrauterine demise in the $S h p 2^{\mathrm{Y} 279 \mathrm{C} / \mathrm{Y2} 29 \mathrm{C}}$ embryos. Because ventricular trabeculation and myocardial compaction are 2 essential steps necessary for a fully functional and competent ventricular wall, we investigated whether NSML mutations affected initiation of trabeculation. Normally, at E10.5, the myocardium begins to thicken, mediating endocardial cell invagination and formation of trabecular protrusions (6). Here, we found that the trabecular area for both $S h p 2^{\mathrm{Y} 279 \mathrm{C} /+}$ and $S h p 2^{\mathrm{Y} 279 \mathrm{C} / \mathrm{YZ279C} \text { embryos was }}$ significantly decreased as compared with WT (Figure 3, D and F), suggesting a defect in onset of myocardial development.

Lineage-specific expression of NSML reveals that SHP2 affects cardiac development primarily through its effects on endocardium. Given that NSML mutations affect both cardiac cushions and ventricular development, we investigated whether the source of the defects stemmed from cell-autonomous or nonautonomous effects of NSML. We crossed inducible NSML mice $\left(\operatorname{Sh} p 2^{\text {in Y279C/+ })}(28)\right.$ to tissue-specific Cre-expressing lines to generate heterozygous NSML expression in neural crest (Wnt1-Cre) (37), endothelium (Tie2-Cre) (38), and myocardium (Nkx2.5-Cre and cTnT-Cre) (39,
40). Despite the fact that adult NSML hearts are associated with myocardial defects (28), we found that nearly all developmental $S h p 2^{\mathrm{Y} 279 \mathrm{C} / \mathrm{+}}$ cardiac phenotypes were recapitulated by endothelialspecific expression of NSML alone (Figure 4A). Indeed, morphometric analysis of endocardial cushion volumes revealed

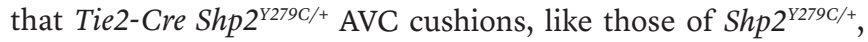
were larger than those of WT (Figure 4B). However, no significant differences were observed in OFT cushion volumes at this stage (data not shown). To assess the effects of NSML mutants on cushion volumes at later developmental stages, we also evaluated P1 neonatal mice. While differences in aortic, pulmonary, mitral, or tricuspid valve volumes in the heterozygous NSML mice appeared to have been resolved by this stage, the homozygous NSML hearts still had significantly enlarged mitral valve volumes (Supplemental Figure 5), suggesting that NSML mutant effects on cushion volumes are likely gene dosage dependent and, for the most part, resolved over time. To support this observation, we conducted aortic regurgitation, insufficiency, and dilation analyses in adult NSML mutant mice. Most of the animals (including WT) had a trace of aortic insufficiency (minor aortic regurgitation or leaking of the aortic valve), which was not of significant consequence. Indeed, the degree of aortic insufficiency seen was 
minimal and did not account for the cardiac malfunction seen in the NSML mutants. We checked to see if there was aortic dilation as well, which may be associated with an enlarged annulus and aortic regurgitation due to inadequate coaptation of the leaflets, but we did not see a difference between the WT and NSML mutants here. Only one animal had a mild mitral valve regurgitation $\left(S h p 2^{\mathrm{Y} 279 \mathrm{C} /+}\right)$, but the rest of the animals had normal mitral valve leaflets (Supplemental Table 3).

In addition, we found that myocardium was also affected by differential NSML expression. The Tie2-Cre Shp2 $2^{\mathrm{Y} 279 \mathrm{C} /+}$ embryos had decreased myocardial trabeculation and thinning of the myocardial compact zone, similar to $\operatorname{Sh} p 2^{\mathrm{Y} 279 \mathrm{C} / \mathrm{+}}$ mice (Figure 4, A and C). Moreover, $c \operatorname{TnT}$-Cre Shp $2^{\mathrm{Y} 279 \mathrm{C} /+}$, but not Nkx2.5-Cre Shp $2^{\mathrm{Y} 279 \mathrm{C} /+}$ myocardial-expressing mice showed decreased wall thickness (Figure 4C), suggesting that a partial cell-autonomous contribution to the NSML phenotype may arise from abnormal signals in the myocardium. In further support of this notion, while we did not observe VSD in Tie2-Cre Shp $2^{\mathrm{Y} 279 \mathrm{C} / \mathrm{+}}$ embryos, this phenotype was apparent in approximately $30 \%$ of $N k x 2.5$-Cre Shp $2^{\mathrm{Y} 279 \mathrm{C} /{ }^{+}}$and $65 \%$ of $c \operatorname{Tn} T$-Cre Shp $2^{\mathrm{Y} 279 \mathrm{C} /+}$ embryonic hearts (Figure $4 \mathrm{~A}$ ), suggesting that the delay in septal closure may be attributable to a cell-autonomous myocardial defect of the NSML mutation. Notably, the more subtle effects of the cell-autonomous contribution to the phenotype by the $N k x 2.5 \operatorname{Cre} \operatorname{Sh} p 2^{\mathrm{Y} 279 \mathrm{C} /{ }^{+}}$can be attributed to a haploinsufficiency of the $N k x 2.5$ gene in myocardium (39). Finally, we also conducted histological analyses in Wnt1-Cre Shp $2^{\mathrm{Y} 279 \mathrm{C} /+}$ embryos; here, we saw no obvious cardiac defects (Figure 4A), despite the presence of craniofacial and skeletal abnormalities of the mice (data not shown), suggesting that LOF of SHP2 phosphatase activity in neural crest does not play a central role in the developing NSML cardiac phenotype. Taken together, our data reveal that NSML mutations of SHP2 affect cardiac development principally through effects on endocardium, although a partial contribution from myocardium is also involved.

Endocardial-specific expression of NSML is sufficient to induce adult-onset cardiac hypertrophy. Given that we recapitulated the majority of the developmental cardiac defects observed in $S h p 2^{\mathrm{Y} 279 \mathrm{C} /+}$ embryos with endocardial-specific expression of NSML alone, we asked whether these mice could also develop adult-onset HCM. Indeed, 16-week-old Tie2-Cre Shp $2^{\text {Y279C/+ }}$ adult mouse hearts were bigger (Figure 5A), with increased heartto-body weight ratios (Figure $5 \mathrm{~B}$ ) and enlarged cardiomyocyte cross-sectional area (Figure 5C), compared with $S h p 2^{+/+}$hearts. Individual isolated cardiomyocytes from Tie2-Cre $\operatorname{Sh} p 2^{\mathrm{Y} 279 \mathrm{C} /+}$ mice were also larger, with significantly increased width, but not length (Supplemental Figure 2, A and B). We also observed a shift in mRNA expression levels from the adult Myh6 ( $\alpha-\mathrm{MHC})$ isoform to the fetal Myh7 ( $\beta$-MHC) isoform in Tie2-Cre Shp $2^{\mathrm{Y} 279 \mathrm{C} /+}$ hearts, indicating onset of pathological hypertrophy (Supplemental Figure 2C). Echocardiography confirmed increased LVPWth and decreased LVDd in both the $S h p 2^{\mathrm{Y} 279 \mathrm{C} /+}$ and the Tie2-Cre Shp $2^{\text {Y279C/+ }}$ adult hearts (Figure 5, D-F; Supplemental Figure 3; and Supplemental Table 2). Remarkably Nkx2.5-Cre Shp $2^{\mathrm{Y} 279 \mathrm{C} /+}$ mice also developed a partial adult-onset cardiac hypertrophy (Figure 5), likely due to the fact that $N k x 2.5$ is expressed in both developing myocardium and endocardium (41). Indeed, we observed no effects on LVPWth or LVDd in the cTnT-Cre Shp $2^{\mathrm{Y} 279 \mathrm{C} /+}$ mice, suggesting that NSML-associated cell-autonomous defects in development do not contribute to the adult-onset NSML hypertrophy phenotype. No effects on hypertrophy were observed in neural crest-specific NSML-expressing mice, consistent with the absence of any developmental defects in this lineage (Figure 5). Taken together, these data confirm that, despite a partial cellautonomous myocardial involvement in the overall NSML phenotype during development, the cardiac hypertrophy in NSML is mediated by aberrant endocardial signaling and, therefore, NSML mutations likely perturb endocardial-to-myocardial crosstalk during development.

NSML mutant expression affects both apoptosis and proliferation in the developing heart. To investigate a developmental mechanism for the endothelial contribution to the adult NSML hypertrophy phenotype, we measured proliferation and apoptosis in $S h p 2^{+/+}$, Shp $2^{\mathrm{Y} 279 \mathrm{C} /+}$, Shp $2^{\mathrm{Y} 279 \mathrm{C} / \mathrm{Y} 279 \mathrm{C}}$, and Tie2-Cre Shp $2^{\mathrm{Y} 279 \mathrm{C} /+}$ endocardial cushions at E14.5. While proliferation significantly increased in all of the NSML genotypes, we observed a concomitant and significant decrease in apoptosis in $S h p 2^{\mathrm{Y} 279 \mathrm{C} /+}, \operatorname{Sh} p 2^{\mathrm{Y} 279 \mathrm{C} / \mathrm{Y} 279 \mathrm{C}}$, and Tie2-Cre Shp $2^{\mathrm{Y} 279 \mathrm{C} /{ }^{+}}$cushions (Figure 6, A-C), likely explaining the enlarged valvular size in NSML and endothelial-specific expressing NSML developing hearts (Figure 3, A-C, and Figure 4B). In contrast, both myocardial proliferation and apoptosis were significantly decreased in $S h p 2^{\mathrm{Y} 279 \mathrm{C} / \mathrm{Y279} \mathrm{C}}$, but not $S h p 2^{\mathrm{Y} 279 \mathrm{C} /{ }^{+}}$and Tie2-Cre Shp $2^{\mathrm{Y} 279 \mathrm{C} /+}$, E14.5 hearts (Figure 6, D-F), likely due to NSML gene dosage-dependent effects of this lineage on the phenotype. Specifically, apoptosis was reduced in both compact and trabecular areas (Supplemental Figure 6A), whereas proliferation was principally affected only in the compact myocardium of this NSML mutant (Supplemental Figure 6B).

NSML leads to downregulation of FOXP1 and NOTCH1 expression. Previously, it was shown that inactivation of Foxp1 induced an embryonic phenotype similar to that observed in NSML-expressing mice, with defects in OFT septation, endocardial cushion development, cardiomyocyte proliferation and maturation, and ventricular septation, leading to midgestation lethality (12). Similarly, NOTCH1 mutants also show impaired trabeculation (42), valve morphogenesis (8), and OFT generation (7). Importantly, both FOXP1 and NOTCH1 regulate intercellular signaling events between the endocardium and the myocardium, either at the level of valve formation and/or myocardial differentiation. Consistently, our data showed that $S h p 2^{\mathrm{Y} 279 \mathrm{C} / \mathrm{+}}, \operatorname{Sh} p 2^{\mathrm{Y279C} / \mathrm{Y} 279 \mathrm{C}}$, and Tie2Cre Shp $2^{\mathrm{Y} 279 \mathrm{C} /+}$ hearts had significantly decreased FOXP1 expression in both valves and myocardium (Figure 7A and Supplemental Figure 7). In addition, FOXP1 transcriptionally repressed genes SOX17, SOX18, and p21 (9) were upregulated, whereas expression of TGF $\beta 2$, an upstream regulator of FOXP1 transcription,

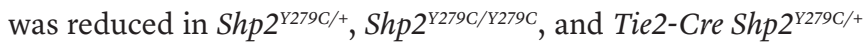
hearts at E14.5 (Figure 7B), confirming downregulation of FOXP1 expression and activity. Surprisingly, no decreases in FGF9 or FGF16 in $S h p 2^{\mathrm{Y} 279 \mathrm{C} /+}, \operatorname{Sh} p 2^{\mathrm{Y} 279 \mathrm{C} / \mathrm{Y} 279 \mathrm{C}}$, or Tie2-Cre $\operatorname{Sh} p 2^{\mathrm{Y} 279 \mathrm{C} /+}$ hearts were detected, suggesting that there may be a feedback mechanism regulating FGF expression in response to disrupted endocardium-myocardium crosstalk in these mice.

Valve-specific NOTCH1 expression was also significantly affected in NSML; Shp $2^{\mathrm{Y} 279 \mathrm{C} /+}, \operatorname{Sh} p 2^{\mathrm{Y} 279 \mathrm{C} / \mathrm{Y} 279 \mathrm{C}}$, and Tie2-Cre Shp $2^{\mathrm{Y} 279 \mathrm{C} /+}$ hearts had significantly decreased NOTCH1 protein 
A
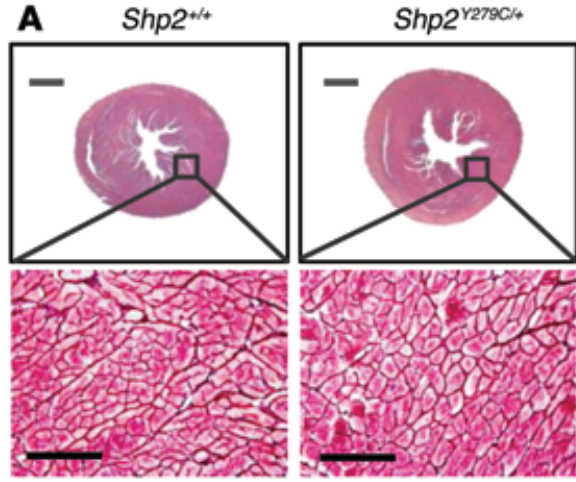

Tie2-Cre Shp2 $2^{\text {r279C/* }}$
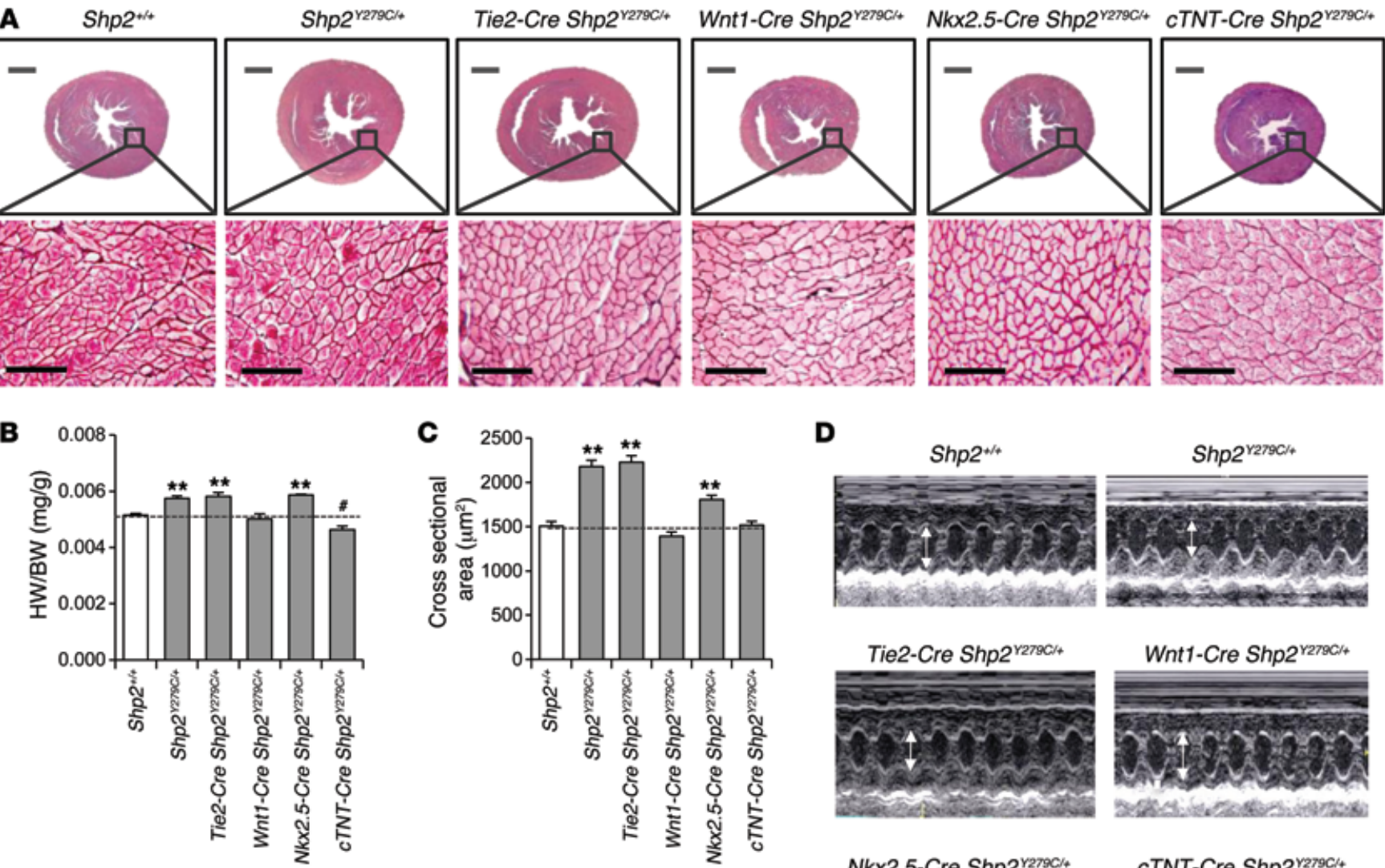

$\mathbf{E}$

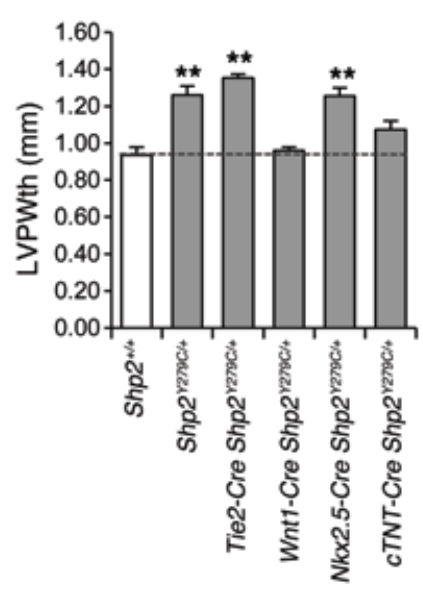

$\mathbf{F}$

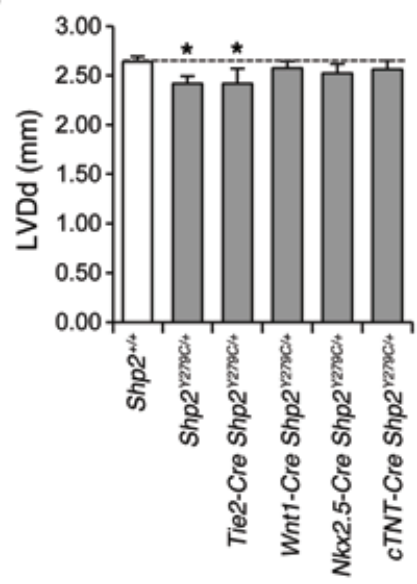

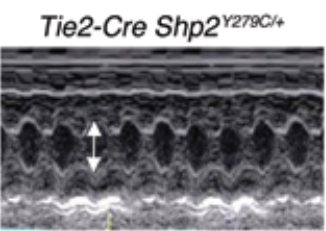

Wnt1-Cre Shp2 $2^{\text {Y279C/* }}$

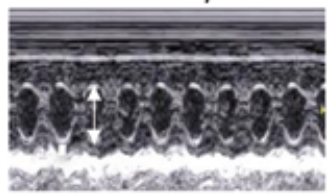

Nkx2.5-Cre Shp2 $2^{\mathrm{r} 279 \mathrm{C} / 4}$
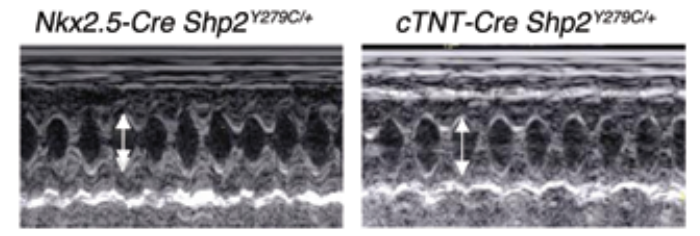

Figure 5. Endothelial expression of NSML alone recapitulates the adult-onset cardiac hypertrophy observed in ubiquitously expressing NSML mice. (A) Transverse cross-section of 16-week-old mouse hearts and corresponding reticulin-stained sections from ubiquitous and lineage-specific expressing NSML mutants. Scale bars: $100 \mu \mathrm{m}$. (B) Heart-to-body weight ratio of 16-week-old mice. (C) Quantification of cardiomyocyte surface area. $n=100-300$ cells counted per group; $n=3$ mice/group. (D) Representative echocardiography of 16-week-old mice. Corresponding echocardiograph analysis of (E) LVPWth and (F) LVDd in diastole. Data represent mean \pm SEM. ${ }^{*} P<0.05 ;{ }^{* *} P<0.01 ;{ }^{*} P<0.05$ (significantly lower than Shp2 ${ }^{+/ *}$ ). $P$ values were derived from 1 -way ANOVA with Bonferroni's post-test when ANOVA was significant.

expression in valves, while no changes were observed in myocardium (Figure 8A and Supplemental Figure 7). Interestingly, Notch1 mRNA expression levels were also significantly decreased, but only in Shp $2^{\mathrm{Y} 279 \mathrm{C} / \mathrm{Y} 279 \mathrm{C}}$ and Tie2-Cre Shp $2^{\mathrm{Y} 279 \mathrm{C} /{ }^{+}}$hearts (Figure 8B), likely a reflection of the dosage-dependent effects of NSML mutations and the requirement for NOTCH1 activity specifically in endocardium. NOTCH1 transcriptionally activated targets HEY2 and PROX1, as well as further downstream targets BMP10, EPHB2, and NRG1, were also downregulated in NSML (Figure 8B). These data indicate that NSML mutations play a central role upstream of FOXP1 and NOTCH1 in perturbing normal cardiac development.

Interestingly, at E10.5, only FGF16 was significantly reduced in Shp $2^{\mathrm{Y} 279 \mathrm{C} /+}$, Sh $p 2^{\mathrm{Y} 279 \mathrm{C} / \mathrm{Y} 279 \mathrm{C}}$, and Tie2-Cre Shp $2^{\mathrm{Y} 279 \mathrm{C} /+}$ embryo hearts (Supplemental Figure 8). Fgf16 null hearts were previously shown to have ventricular wall thinning and poor trabeculation (43), both of which were apparent in NSML embryos at E10.5. No 
A

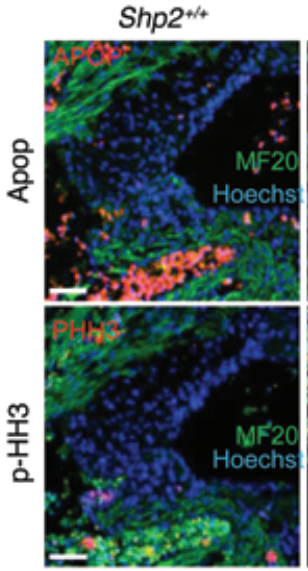

D

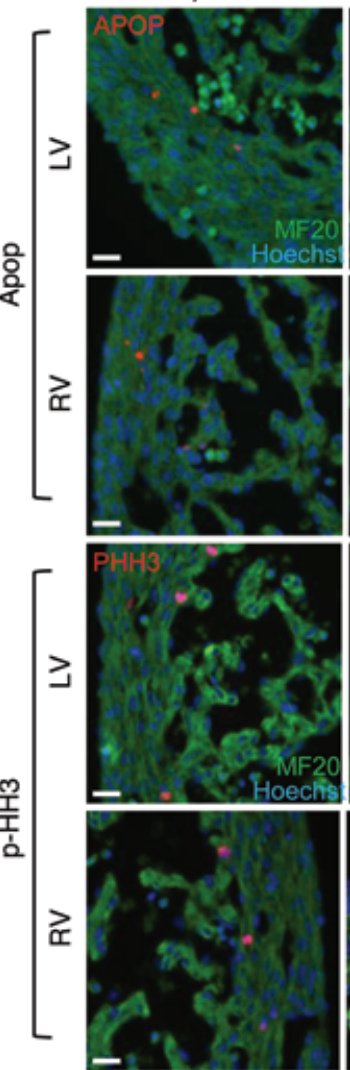

Shp2 $2^{\text {refoc/4 }}$

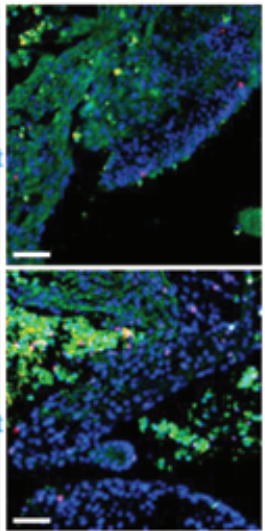

Shp2 $2^{\text {r279C/4 }}$
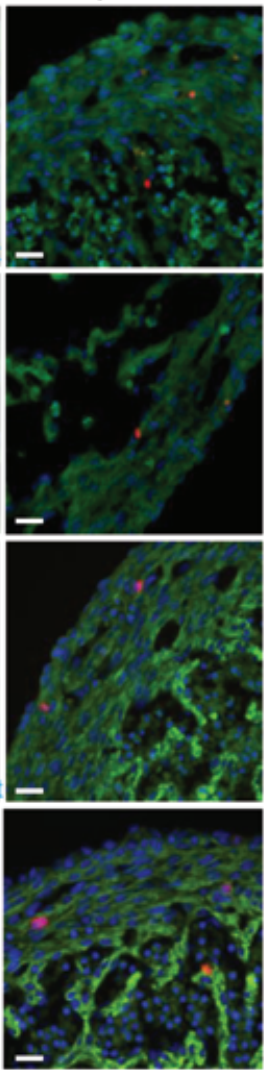

Shp2 $2^{\text {r279C/2799 }}$

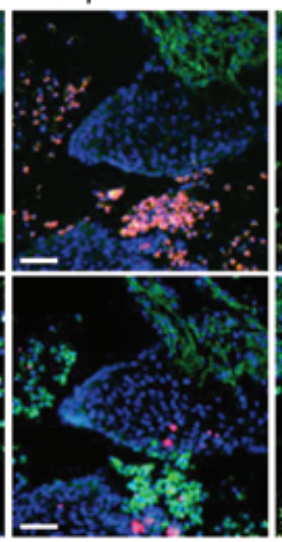

Shp2 $2^{\text {r279Crrac }}$
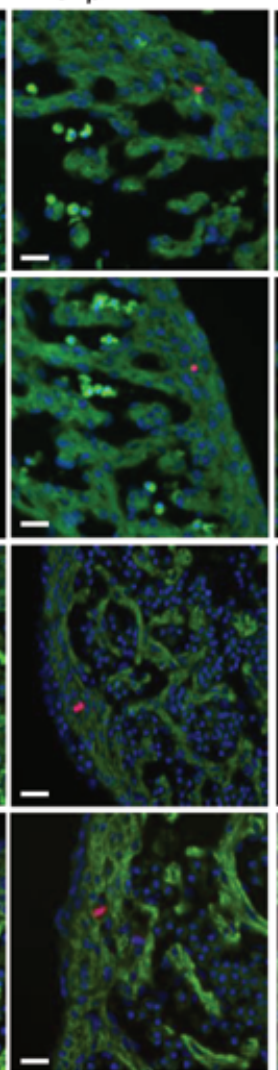

Tie2-Cre Shp2 $2^{\text {re79c/t }}$

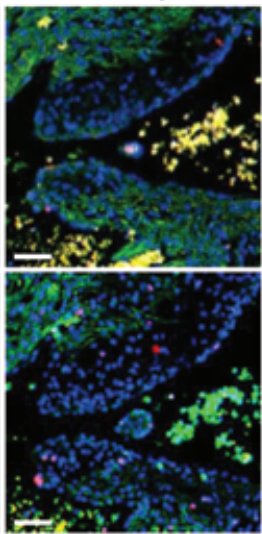

Tie2-Cre Shp2 ${ }^{\text {rez9c/t }}$
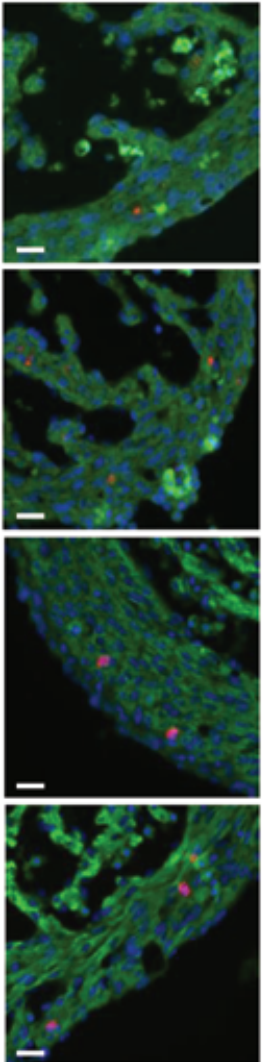

B
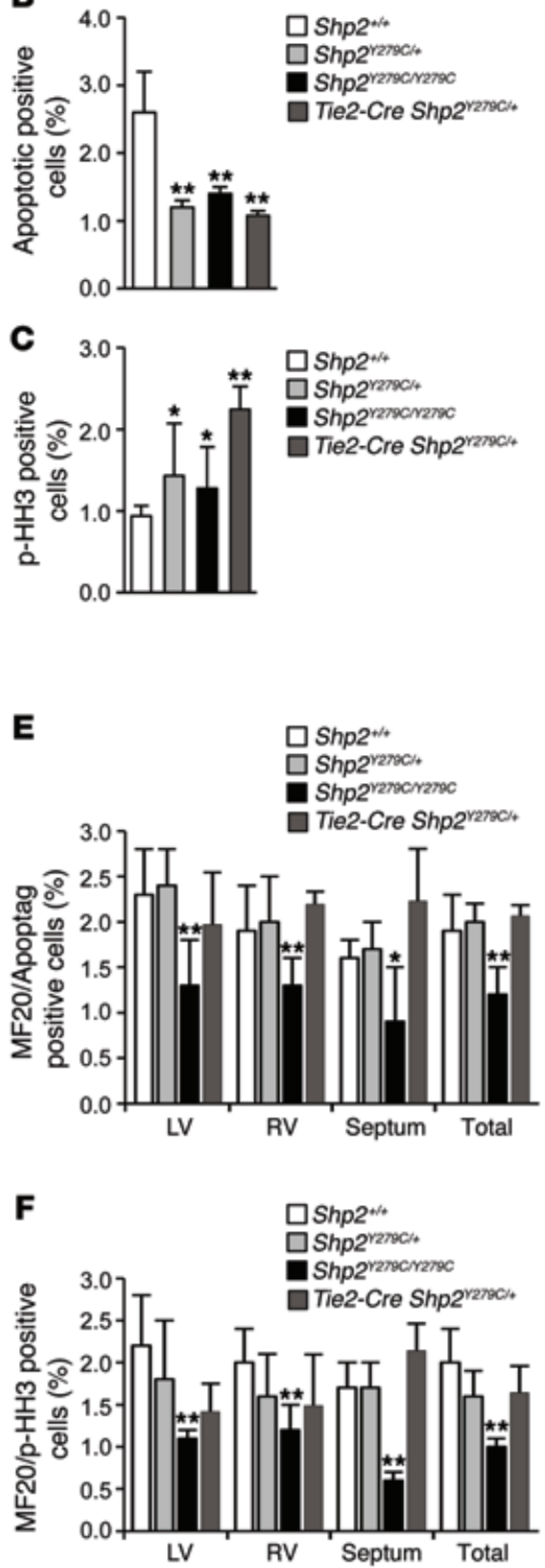

Figure 6. NSML expression affects both apoptosis and proliferation in the developing heart. (A) Immunofluorescence staining of E14.5 embryo hearts representative of endocardial cell apoptosis and proliferation using Apoptag (top panels) to mark apoptotic cells (red) or p-HH3 (lower panels) to mark proliferation (red). Additional double-staining for MF20 was used to identify myocardium (green), and Hoechst stain was used to mark the nuclei, and in this case, to delineate the mitral valves. Scale bars: $50 \mu \mathrm{m}$. Quantification of endocardial (B) apoptosis and (C) proliferation is demonstrated from $n=4$ embryos per group per analysis, with counts equal to or greater than 1000 cells/embryo/defined area. (D) Immunofluorescence staining of myocardial cell apoptosis and proliferation using Apoptag (upper panels) to mark apoptotic cells (red) or p-HH3 (lower panels) to mark proliferation (red) in left ventricle or right ventricle. Additional double-staining for MF20 was used to identify myocardium (green), and Hoechst stain was used to mark the nuclei. Scale bars: $50 \mu \mathrm{m}$. p-HH3, phospho-histone H3. Quantification of myocardial (E) apoptosis and (F) proliferation are demonstrated in left ventricle, right ventricle, septum, and total myocardium, with $n=4$ embryos per group per analysis, with counts equal to or greater than 1000 cells/embryo/defined area. All data are expressed as mean $\pm \mathrm{SEM}$. ${ }^{*} P<0.05 ;{ }^{*} P<0.01$. $P$ values were derived from 1-way ANOVA with Bonferroni's post-test when ANOVA was significant.

other FOXP1 or NOTCH1 pathway-related genes were affected at this early stage (Supplemental Figure 8).

NSML developing hearts have elevated signaling effects driving hypertrophy. AKT/mTOR activity is upregulated in adult $S h p 2^{\mathrm{Y} 279 \mathrm{C} /+}$ hearts, the inhibition of which normalizes NSML-associated HCM $(28,29)$. Since AKT functions as a negative upstream regulator of both FOXP1 and NOTCH1 signaling, we investigated whether AKT could play a central role in NSML-associated cardiac pertur- 
A

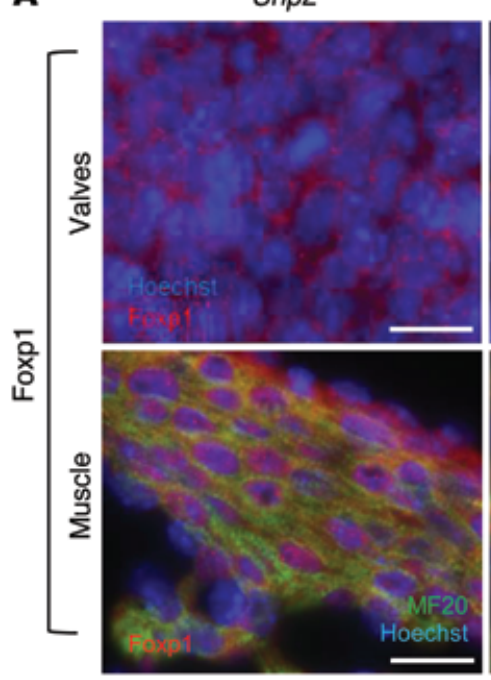

Shp2 $2^{\text {r2790/4 }}$
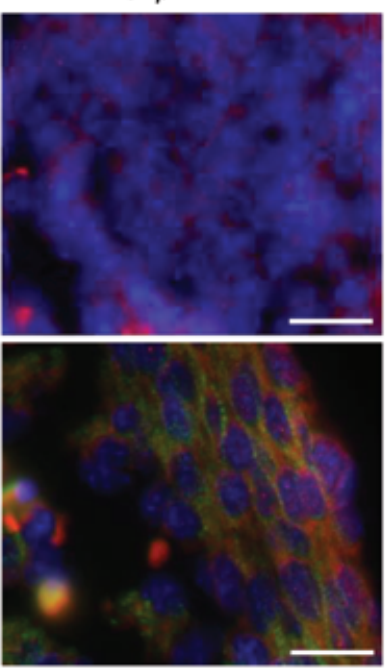

Shp2 $2^{\text {ras9crarac }}$
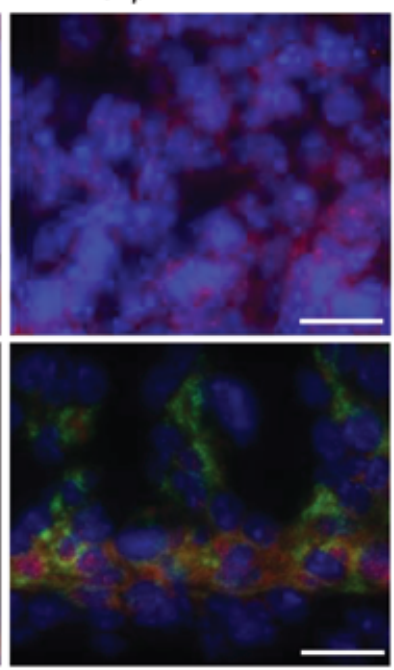

Tie2-Cre Shp2 $2^{\mathrm{rz} 9 \mathrm{C} /+}$

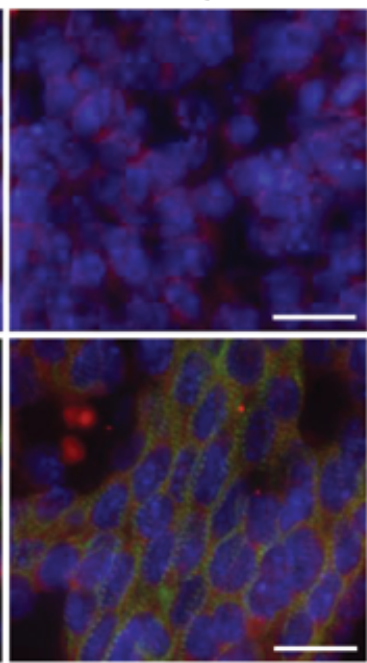

B

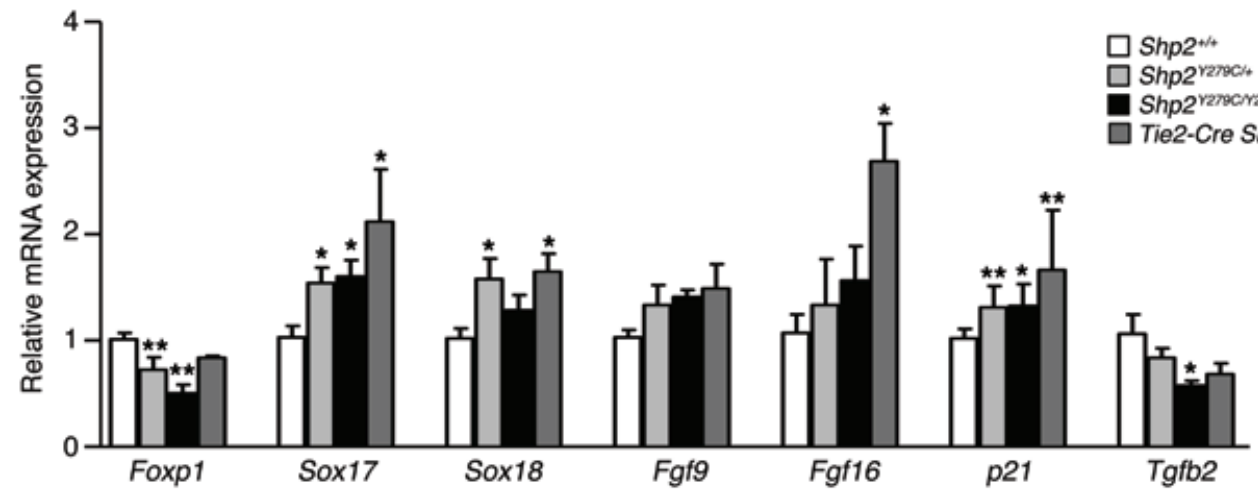

Figure 7. LOF mutations in PTPN11 lead to downregulation of FOXP1. (A) Immunofluorescence staining of E14.5 embryo hearts representative of endocardial (upper panels) and myocardial expression (lower panels) of FOXP1 expression (red) at high magnification. Scale bars: $20 \mu \mathrm{m}$. Additional doublestaining for MF20 was used to identify areas with myocardium (green, lower panels), and Hoechst stain was used to mark the nuclei (upper and lower panels) and to demarcate the valves (upper panels). (B) mRNA expression in embryo hearts at E14.5 quantified by qPCR showing a decrease in Foxp1 gene expression and signaling in NSML hearts as compared with control $\left(S h p 2^{+++}\right) . n=6$ embryos per group. Data represent mean \pm SEM. ${ }^{*} P<0.05$; ${ }^{* *} P<0.01$. $P$ values were derived from 1-way ANOVA with Bonferroni's post-test when ANOVA was significant.

bations during development. Indeed, analysis of embryonic hearts at E14.5 revealed a gene dosage-dependent increase in AKT phosphorylation (Figure 9A), consistent with the observed decrease in FOXP1 and NOTCH1.

Interestingly, it has been demonstrated that overexpression of AKT increases nuclear localization of NFATc1 in osteoclastogenesis (44), suggesting that increased AKT activity can induce activation of NFAT-associated HCM gene transcription. Moreover, FOXP1 plays a dichotomous role with NFAT in the transcriptional regulation of HCM; decreased FOXP1 leads to decreased expression of genes required to maintain normal heart function and to increased activity of NFAT, which induces HCM-associated genes (45). Therefore, imbalance toward increased NFAT activity, either by increased AKT activity and/or decreased FOXP1 expression, leads to upregulation of genes that mediate cardiomyocyte hypertrophy (45). We assessed NFAT activity in NSML embryos by measuring its phosphorylation, indicative of nuclear exclusion and inactivity, and observed a similarly significant gene dosage-dependent decrease in phosphorylated NFAT in NSML hearts (Figure 9B). We confirmed NFAT activation by assessing its nuclear localization, indicative of transcriptional activation, and found it to be increased in all NSML mutant genotypes (Figure 9C). Together, these data indicate that, in addition to decreased NOTCH1 and FOXP1, NSML mutations also induce NFAT activation to mediate hypertrophy.

\section{Discussion}

The molecular mechanisms regulating cardiac development and morphogenesis are dependent on crosstalk between multiple cell lineages, the aberrant regulation of which can induce the onset of CHD and phenotypes such as HCM. Here, using our SHP2 knockin mouse model $\left(\operatorname{Sh} p 2^{\mathrm{Y} 279 \mathrm{C} /+}\right)$, we sought to determine whether NSML-associated HCM stems from aberrant cardiac developmental processes. Our data show that embryonic NSML hearts had diminished trabeculation and valvular hyperplasia, defects recapitulated in endocardial-specific, but not myocardial-specific or neural crest-specific NSML mice. Indeed, endocardial-specific expression of NSML was sufficient to induce adult-onset cardiac 
A

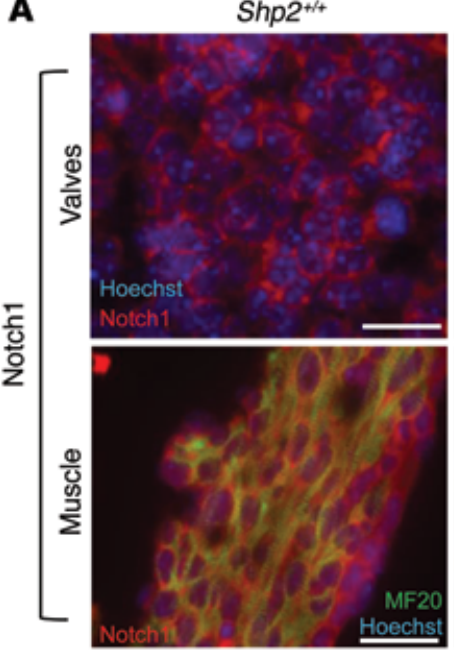

Shp2 $2^{\text {Y279C/4 }}$
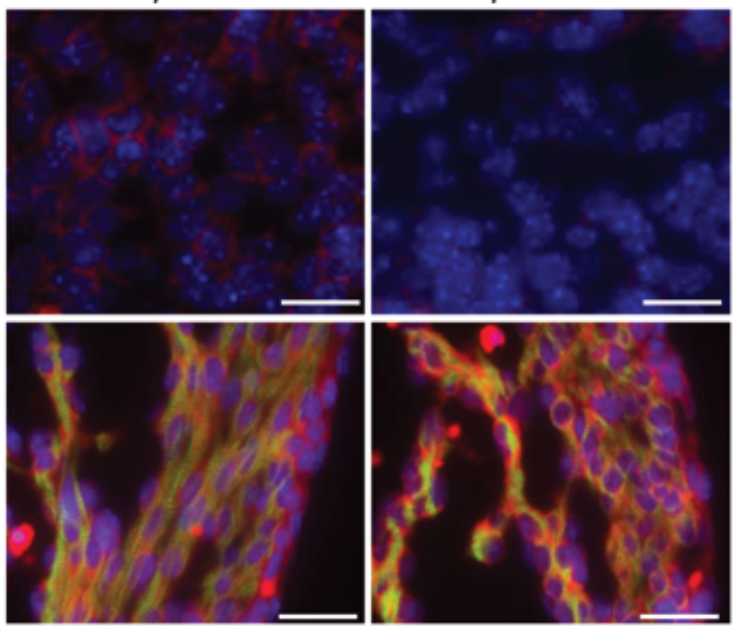

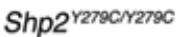

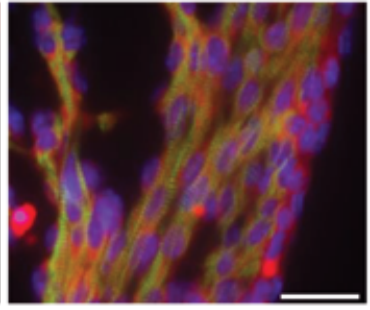

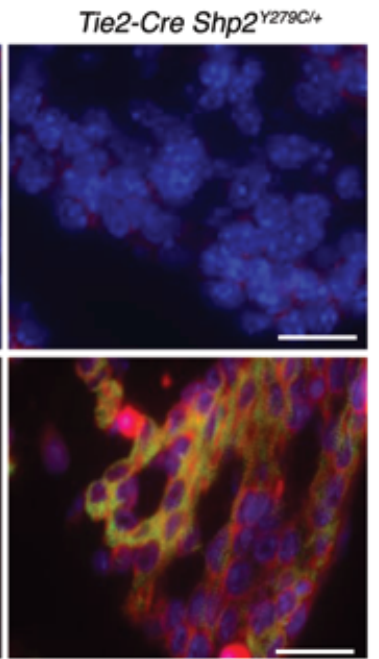

$\mathbf{B}$

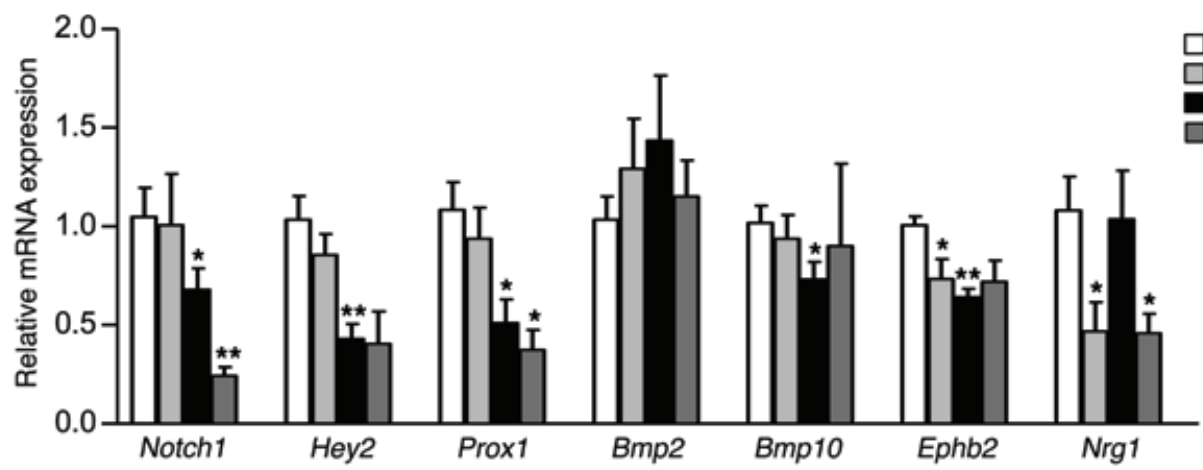

Figure 8. LOF mutations in PTPN11 lead to downregulation of Notch1. (A) Immunofluorescent staining of E14.5 embryo hearts representative of endocardial (upper panels) and myocardial expression (lower panels) of Notch1 expression (red) at high magnification. Scale bars: $20 \mu \mathrm{m}$. Additional doublestaining for MF20 was used to identify areas with myocardium (green, lower panels), and Hoechst stain was used to mark the nuclei (upper and lower panels) and to demarcate the valves (upper panels). (B) mRNA expression in embryo hearts at E14.5 quantified by qPCR showing a decrease in Notch1 gene expression and signaling in NSML hearts as compared with control $\left(S h p 2^{+/+}\right) . n=6$ embryos per group. Data represent mean $\pm S E M ;{ }^{*} P<0.05 ;{ }^{* *} P<0.01$. $P$ values were derived from 1-way ANOVA with Bonferroni's post-test when ANOVA was significant.

hypertrophy. In addition, NSML embryos developed VSDs, a phenotype reproduced only in myocardial-specific NSML hearts. Interestingly, myocardial-specific NSML developmental defects resolved and were likely a consequence of developmental delay due to abnormal communication between endocardium and myocardium, particularly as these lineage-specific mice did not develop adult-onset HCM. Therefore, our results demonstrate what we believe to be a novel role for SHP2 in regulating both cell-autonomous and nonautonomous effects in the developing heart through regulation of the pathways that control endocardial-to-myocardial crosstalk (Figure 10).

Alignment and ventricular septal wall defects can result from abnormal looping and/or remodeling of the primitive heart tube into a multi-chambered, fully functional heart. Indeed, proper positioning of internal organs, and consequent partitioning of the heart and connection to the vasculature, is dependent on multiple signals establishing left-right (LR) organ asymmetry. Perturbations of LR patterning, leading to minor situs defects, are thought to be responsible for many aspects of CHD (46). Recently, work on both zebrafish and xenopus embryo models expressing SHP2 mutants demonstrated that SHP2 plays an important role in LR asymmetry. Specifically, in zebrafish, NS and NSML mutations of SHP2 resulted in defective leftward heart displacement and randomization of LR asymmetry markers (47). Similarly, in xenopus, NS SHP2 mutants showed smaller hearts that failed to undergo complete looping, with improper chamber formation and delayed morphogenic movements mediated by defective cardiac actin fibers and F-actin deposition (48). Together, these studies suggest that SHP2 is fundamental to cardiac looping, the failure of which might be responsible for the alignment defects and VSD phenotypes we observe in our NSML mouse model.

Given that we showed a variably penetrant alignment defect affecting the aorta in our NSML mouse model and that mice with neural crest-specific deletion of SHP2 previously demonstrated cardiac defects, including persistent truncus arteriosus and anomalies of aortic arch (49), we expected a similar phenotype in the neural crest-expressing NSML mice. However, despite abnormal craniofacial effects indicative of neural crest origins, we did not observe any obvious aberrant cardiac phenotypes, suggesting that LOF of SHP2 activity does not affect neural crest-derived development of the heart. Similarly, murine neural crest-specific expression of NS does not give a cardiac phenotype (50). Indeed, 
A

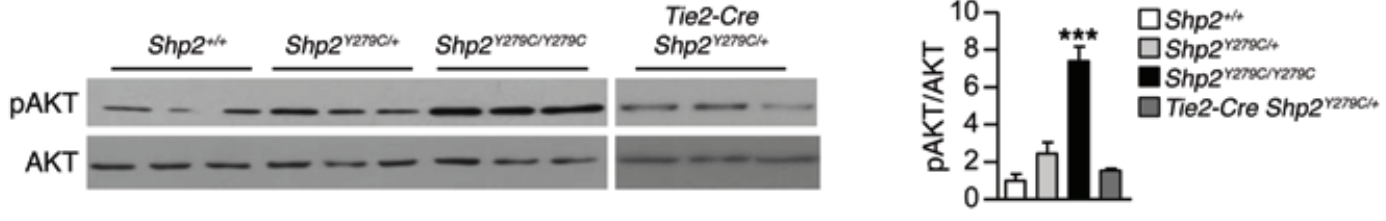

B
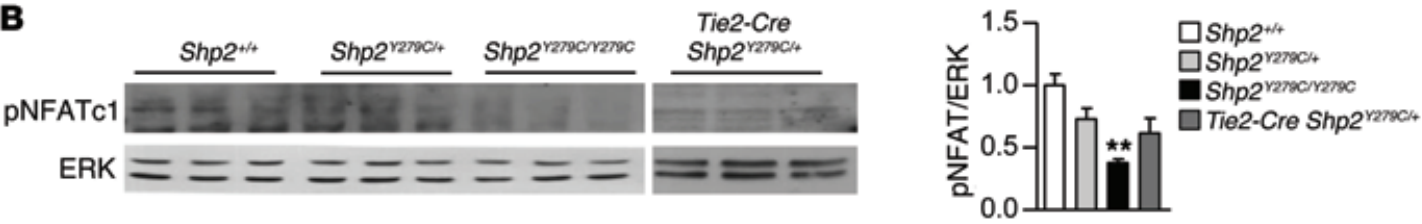

C
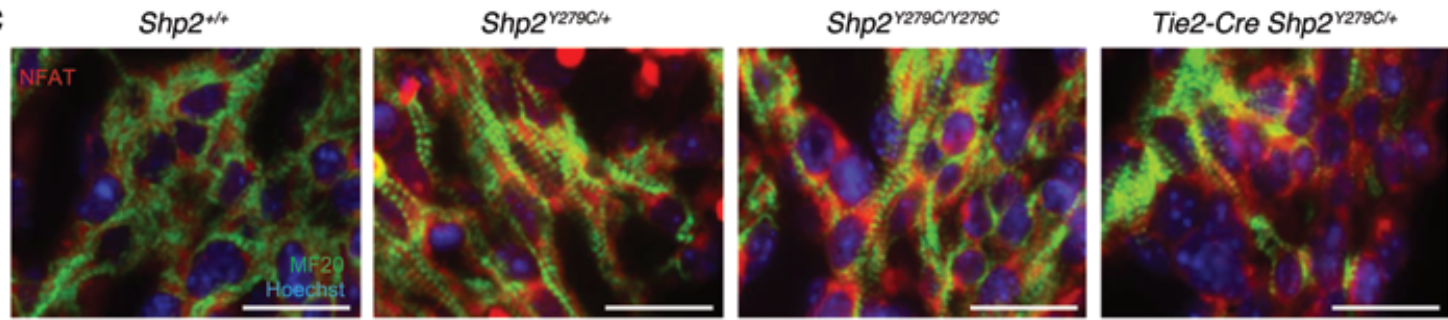

Figure 9. NSML mutations increase AKT activity in embryos, concomitant with upregulation of NFAT activity. Protein lysates isolated from E14.5 embryo hearts were immunoblotted with (A) anti-phospho-AKT and (B) anti-NFATc1, as indicated, followed by anti-AKT or anti-ERK to control for loading, respectively. Quantification of Western blots from $n=3$ independent experiments is indicated to the right of $\mathbf{A}$ and $\mathbf{B}$, respectively. Data represent mean \pm SEM. ${ }^{* *} P<0.01 ;{ }^{* *} P<0.001$. $P$ values were derived from 1-way ANOVA with Bonferroni's post-test when ANOVA was significant. (C) Immunofluorescent staining representative of myocardial NFATc1 expression and activity through increased translocation of NFATc1 in the nucleus (red). Additional doublestaining for MF2O was used to identify areas with myocardium (green), and Hoechst stain was used to mark the nuclei. Scale bars: $20 \mu \mathrm{m}$.

taken together, these data imply that SHP2 contribution to cardiac development by neural crest cell derivatives likely depends on noncatalytic, phosphatase-independent functions of SHP2.

The existence of potential noncatalytic functions of SHP2 might also help explain the major conundrum posed by diseasecausing SHP2 mutations; despite having opposing enzymatic functions, SHP2-associated NS and NSML mutations cause similar disease phenotypes. Here too, we showed that NSML mutations resulted in developmental cardiac defects similar to those previously observed in NS, including thickened valves, variably penetrant atrioventricular septal defects, and myocardial wall thinning (50). Moreover, we found catalytically inactive NSML mice to be viable, suggesting that noncatalytic functions of the enzyme are at least necessary and sufficient to proceed through development. In this regard, however, we cannot exclude the possibility that the slight, albeit minimal, phosphatase activity in NSML mice is sufficient to overcome the embryonic lethality observed in Shp2deleted mice; determination of the functional and mechanistic consequences for the phosphatase-independent roles of SHP2 are currently underway in our laboratory.

Another possibility for the similar phenotypes in GOF NS and LOF NSML patients is that the SHP2 mutations themselves act on different stages of cardiac development and/or through different signaling pathways. For example, mice bearing an EGFR-hypomorphic allele (wa2/wa2) exhibit semilunar valve enlargement resulting from overabundant mesenchymal cells, a phenotype further enhanced by heterozygous deletion of SHP2 (51). Likely, this phenotype is a consequence of enhanced endocardial cushion cell proliferation mediated through EGF's ability to inhibit BMP- mediated activation of SMAD1/5/8 in the AVC and OFT (52). In contrast, GOF of SHP2 in NS, which also leads to increased valve size, facilitates EndoMT during valvulogenesis through upregulation of ERK signaling (50). The importance of ERK activity in mediating abnormal valve development in NS was further demonstrated when transgenic SHP2 ${ }^{\mathrm{Q} 79 \mathrm{R}}$ mice were crossed to homozygous ERK1 deleted mice, rescuing the endocardial cushion defects (53). Interestingly, and despite the fact that the cardiac defects in NS mice (50), as in our NSML model herein, are recapitulated by endocardial-specific expression of the mutant, our data indicate that the causal signaling mechanisms responsible for the NSML phenotypes differ from those found in NS. Specifically, the LOF NSML mutations in SHP2 increase valve size, cause myocardial thinning, and induce VSD through aberrant endocardial-to-myocardial crosstalk mediated by increased AKT, not ERK, signaling.

Indeed, our data indicate that the aberrant AKT activity in NSML embryos also mediates decreased downstream signaling of FOXP1/FGF and NOTCH1/EPHB2, 2 pathways necessary for reciprocal crosstalk between developing endocardium and myocardium. Previously, it was demonstrated that NOTCH1 is a direct target of p53 in human keratinocytes (54) and epithelial cells (55). Increasing AKT signaling, which drives MDM2-mediated p53 degradation (56), may therefore cause decreased NOTCH1 expression in NSML. Indeed, the NOTCH pathway has been identified as a crucial cell-fate regulator in the developing heart, with region-specific endocardial NOTCH activity responsible for the patterning and morphogenesis of cardiac chambers and valves through regulatory interaction with multiple signals, including in myocardium (57). It has also been shown to regulate cardiomyo- 


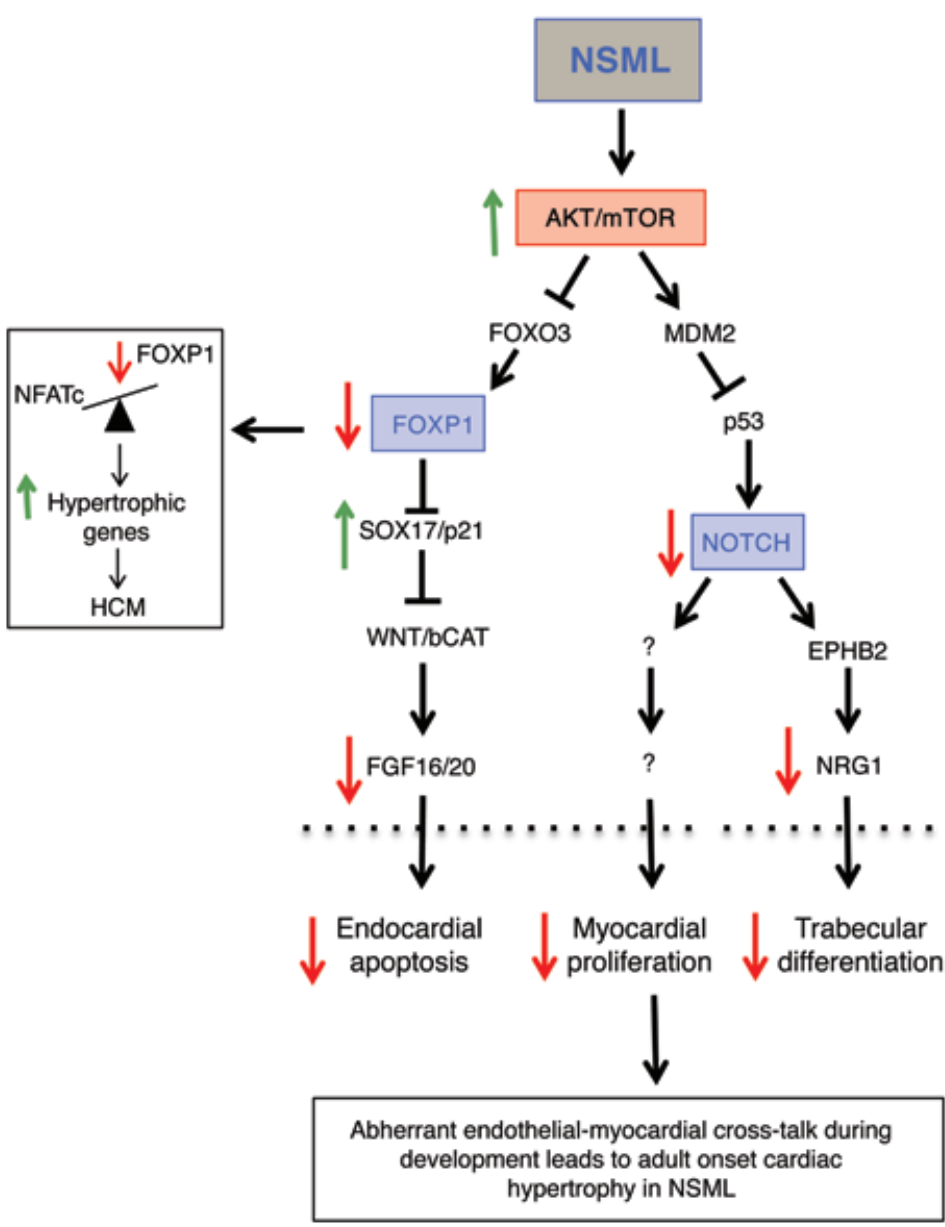

Figure 10. Proposed model: aberrant regulation of NSML mutants in developing hearts leads to adult-onset cardiac hypertrophy. NSML mutations affect AKT signaling in the developing endocardium, decreasing the transcriptional regulation of FOXP1 and NOTCH, 2 integral signaling pathways involved in the endocardialto-myocardial crosstalk necessary for proper cardiac development. This abnormal regulation results in enlarged and amorphic heart valves, decreased trabeculation and ventricular development, and cardiac developmental delay in the embryos. Ultimately, these abnormal signaling events that occur during development are responsible for adult-onset cardiac hypertrophy.

trophy via cell-autonomous mechanisms driven by AKT signaling. In vitro, deletion of SHP2 in neonatal rat ventricular myocytes (NRVM) leads to increased cardiomyocyte cell size (28). Similarly, expression of the NSML SHP2 ${ }^{\mathrm{D} 510 \mathrm{E}}$ mutant in NRVM (29) and in P19CL6 cells (59) also resulted in increased cell size. Moreover, in vivo cardiomyocytespecific transgenic overexpression of the SHP2 ${ }^{\mathrm{Q} 510 \mathrm{E}}$ NSML mutant using a $\beta$-MHC cardiomyocyte promoter led to a severe early onset cardiac hypertrophy mediated by increased AKT/mTOR activity (29). In addition, our previous work (28) and the data herein support a central role for AKT/mTOR in NSML-associated cardiac hypertrophy, with increased AKT activity observed in both embryo and adult NSML hearts.

Further support for a cell-autonomous role for SHP2 in HCM was demonstrated here by the fact that we observed increased NFAT activity in NSML embryos. Overexpression of a constitutively activated NFATc4 mutant under the control of the cardiomyocyte-specific Myh 6 ( $\alpha$-MHC) promoter was previously shown to result in cardiac hypertrophy (60). Moreover, NFATc3-deleted mice demonstrate attenuated

cyte proliferation and differentiation during ventricular chamber development and is required for coronary vessel specification (57).

Similarly, FOXP1 is a direct transcriptional target of Forkhead box transcription factor class O (FOXO) (58). Here, increasing AKT activity, which induces phosphorylation and inactivation of FOXO, may also be responsible for the decrease in FOXP1 expression in NSML. Alternatively, or in parallel, downregulation of TGF- $\beta$, as seen in the NSML mutant hearts, could also be responsible for the decrease in FOXP1 expression. Interestingly, Tie2-mediated endocardial expression of NSML alone was sufficient to reduce both endocardial and myocardial expression of FOXP1 (Figure 7), further implicating the importance of the integral crosstalk functions between endocardium and myocardium in cardiac development and NSML. While this is likely orchestrated by a panoply of paracrine/autocrine effects, the precise mechanisms and signals for the crosstalk remain unknown. Here, we show that it is a combined aberrant regulation of both NOTCH1 and FOXP1 that likely causes the cardiac developmental phenotypes in NSML. Importantly as well, while our data strongly suggest that the adult HCM phenotype arises from effects of NSML-associated SHP2 expression principally stemming from the developing heart, we cannot exclude the possibility that SHP2 may (also) play a role in adult endothelial and/or myocardial lineages, possibly as a regulator of adult heart homeostasis.

Importantly, several studies have suggested that abnormal regulation of SHP2 can drive adult-onset cardiomyocyte hyperresponses to diverse hypertrophic stimuli, suggesting that NFAT activation induces the hypertrophic response in myocardium (61).

While both AKT and NFAT activation in NSML are likely responsible for the cell-autonomous contribution to cardiomyocyte hypertrophy, we found that important non-cell-autonomous mechanisms also exist. Indeed, the most striking observation in our study is the fact that the endocardial-specific expression of NSML alone was sufficient to induce adult-onset cardiac hypertrophy, suggesting that SHP2 regulation of endocardial-to-myocardial crosstalk is essential for cardiac muscle development. Endocardial-to-myocardial crosstalk may involve upregulation of paracrine factors delivered by the endocardium to the myocardium to perturb cardiac homeostasis, factors that may induce growth factor receptor signaling pathways involving SHP2. As an example, it was previously shown that transgenic mice overexpressing related transcriptional enhancer factor-1 (RTEF-1) in an endothelial-specific manner develop significant cardiac hypertrophy after transverse aortic constriction as a result of increased VEGF-B production (62). While we did not detect an increase in Vegfb mRNA expression in our model (data not shown), we cannot rule out the possibility that other, perhaps more SHP2-dependent, endocardial paracrine factors might be involved in NSML cardiac hypertrophy in this way.

Additionally, our data may suggest a collective mechanism for the developmental delay and the spectrum of observed NSML- 
associated abnormalities, with early embryonic developmental defects driving adult cardiac hypertrophy. The early demise of a large proportion of $S h p 2^{Y 279 C / Y 279 C}$ embryos and the associated decrease in ventricular wall thickness in both $S h p 2^{\mathrm{Y} 279 \mathrm{C} /+}$ and

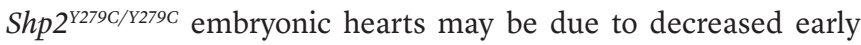
myocyte proliferation and cardiac insufficiency, while abnormal survival and retention of embryonic precursor myocytes might later contribute to hypertrophic cardiac growth in survivors. Indeed, the diminished embryonic wall thickness, as a consequence of perturbed endocardial-to-myocardial crosstalk, might increase the work load on the heart wall and therefore, by compensatory mechanisms, result in cardiac hypertrophy. Interestingly, developmental valve defects can also lead to cardiac hypertrophy, likely through effects on ventricular work load. For example, endothelial-specific deletion of ADAM17 or HB-EGF results in aberrant compartmentalization of versican and increased deposition of collagen in semilunar valves, affecting valvular maturation and increasing the size of valves. Moreover, these defects are associated with decreased postnatal viability, progressive cardiomegaly, and systolic dysfunction $(63,64)$.

Taken together, our data suggest that NSML mutations induce AKT hyperactivation during cardiac embryonic development, perturbing the expression of essential genes involved in the reciprocal crosstalk between endocardium and myocardium, thereby inducing HCM through downregulation of FOXP1 and NOTCH1 signaling pathways and upregulation of NFAT activity (Figure 10).

\section{Methods}

Mice. Sh $p 2^{\text {inY279C/+ }}$ and $S h p 2^{Y 279 C /+}$ mice were described previously (28), and are now available from The Jackson Laboratory (stock no. 026759). Here, timed matings of heterozygous $\operatorname{Sh} p 2^{\mathrm{Y} 279 \mathrm{C} /+}$ males and females were conducted, and the date of observation of a vaginal plug was set as E0.5 by convention. For tissue-specific expression of the NSML allele, males carrying the Cre recombinase under the control of a tissue-specific promoter were crossed to female $\operatorname{Sh} p 2^{\text {inY279C/+ }}$ mice. Tie2Cre (stock no. 004128) and Wnt1-Cre (stock no. 022501) mice were obtained from The Jackson laboratory. Nkx2.5-Cre mice were provided by Robert J. Schwartz (University of Houston, Houston, Texas, USA). cTNT-Cre mice were provided by William T. Pu (Children's Hospital Boston, Boston, Massachusetts, USA). Only male progeny were used for the experiments herein, and all mice were maintained on outbred C57BL6/J backgrounds, backcrossed for more than 10 generations.

Histology. Following maternal sacrifice, placentae, embryos at the indicated stages (E10.5 or E14.5) or neonates (P1) were retrieved and dissected in ice-cold PBS, fixed in Bouin's reagent for morphometric analyses or in 4\% PFA for immunohistochemical staining, and paraffin embedded. For morphometric analyses, serial sections through the entire placenta or heart $(5 \mu \mathrm{m})$ were stained with H\&E. Endocardial cushion volumes, total volumes of trabecular sheets relative to ventricular volumes, and ventricular wall thicknesses were measured by quantifying the volumes or thicknesses from each embryonic section using Image 1.41 software (NIH) and then adding the individual values together to reconstruct the tissue. For immunohistochemical staining, following antigen retrieval with heat treatment in unmasking solution (Vector Laboratories), sections from E14.5 embryo hearts were stained with anti-FOXP1 (Cell Signaling Technology), anti-NOTCH1 (Cell Signaling Technology), and anti-NFAT (Santa Cruz Biotechnology Inc.) to identify expression levels of these proteins in valves and myocardium; anti-MF20 (DSHB), an anti-myosin heavy chain antibody, to identify myocytes; p-HH3 (Millipore), an anti-phospho-histone H3 antibody, to identify proliferating cells; or Apoptag Red (Millipore) to identify apoptotic cells. Nuclei were counterstained with Hoechst dye (Molecular Probes). Mitral valves and left ventricular tissue near the apex were used to assess proliferation and apoptosis in endothelium and myocardium, respectively; demarcation of the trabecular and compact zones was assessed by our rodent histopathologist, Roderick Bronson, and Kyu-Ho Lee. Sections were digitally photographed with a Zeiss AxioImager M2 or a Keyence BZ-X710 for cell counting and analysis. Atrioventricular endocardial cushion cells were identified by morphology and MF20 negativity. Images from immunohistochemical sections were taken at the same intensity across all genotypes and were analyzed using Keyence BZ-X Analyzer Software.

For adult mice, 12- or 16-week-old adult hearts, as indicated, were flushed with PBS, perfusion fixed in Bouin's reagent, and paraffin embedded. Sections ( $5 \mu \mathrm{m})$ were stained with $\mathrm{H} \& \mathrm{E}$, Masson-trichrome, collagen, or reticulin at the Rodent Histopathology Core by Roderick Bronson. Body weights, dry heart and lung weights, and tibia lengths were assessed upon sacrifice of the animals. Cardiomyocyte cross-sectional areas, with centrally located nuclei to ensure the same plane of sectioning, were measured using ImageJ. From 200 to 500 cells were measured for each genotype. Perivascular and interstitial cardiac fibrosis measurements were assessed by the Keyence Analyzer software; similar sections were imaged and measured per genotype at $\times 40$.

Quantitative real-time PCR. RNA was isolated from whole hearts using the RNeasy Mini Kit (QIAGEN). Quantitative real-time PCR was performed with SYBR Green (Applied Biosystems) using an Applied Biosystems 7900 Real-Time PCR System. Gene expression analyses for the indicated genes were carried out according to the manufacturer's instructions. Primer sequences and conditions are provided in Supplemental Table 4. Each measurement was obtained using 6 embryos per genotype for E14.5 and 3 embryos per genotype for E10.5, and each sample was measured in triplicate. Data were quantified using the comparative CT method, with TBP and RPL13 expression levels serving as controls.

Echocardiography. Transthoracic echocardiography was conducted on nonanesthetized animals as described previously (28) with a 13-MHz probe (Vivid 7, GE Medical Systems) or VisualSonics Vevo 770 high-frequency ultrasound rodent imaging system. GE Medical Systems or VisualSonics Vevo 770 software was used for data acquisition and subsequent analysis. Hearts were imaged in the 2D parasternal short-axis view, and an M-mode echocardiogram of the midventricular region was recorded at the level of the papillary muscles. Calculations of cardiac anatomic and functional parameters were carried out as described previously (28).

Aortic regurgitation, dilation, and insufficiency measures. A highfrequency ultrasound scanner (VisualSonics Vevo 2100) with a 50-MHz transducer (VisualSonics MS550S) was used to assess aortic regurgitation, dilation, and insufficiency, as previously described (65). Briefly, following isoflurane anesthesia (1\% in oxygen) via a mask, color- and pulse wave-Doppler flow spectra were recorded at the aortic valve leaflets. Doppler spectra acquisition and analysis were performed in a genotype-blinded manner by Saumya Das (cardiologist) and Federico Damilano (molecular biologist with expertise in mouse aortic regurgitation). 
Biochemical analyses. Whole hearts from E14.5 Shp2 $2^{+/+}, \operatorname{Sh} p 2^{\mathrm{Y} 279 \mathrm{C} /+}$, $\operatorname{Sh} p 2^{\mathrm{Y} 279 \mathrm{C} / \mathrm{Y} 279 \mathrm{C}}$, and Tie2-Cre Shp $2^{\mathrm{Y} 279 \mathrm{C} /+}$ littermate embryos were dissected in PBS and immediately frozen in liquid $\mathrm{N}_{2}$. Whole-cell lysates were prepared by homogenizing the tissue in radioimmunoprecipitation (RIPA) buffer (25 mmol/l Tris- $\mathrm{HCl}$ [pH 7.4], $150 \mathrm{mmol} / \mathrm{l} \mathrm{NaCl}$, 0.1\% SDS, $1 \%$ NP-40, 0.5\% sodium deoxycholate, 5 mmol/1 EDTA, $1 \mathrm{mmol} / \mathrm{l} \mathrm{NaF}, 1 \mathrm{mmol} / \mathrm{l}$ sodium orthovanadate, and a protease cocktail) at $4^{\circ} \mathrm{C}$, followed by clarification at $14,000 \mathrm{~g}$. Proteins were resolved by SDS-PAGE and transferred to PVDF membranes. Immunoblots were performed, following the manufacturer's directions, with anti-AKT (Santa Cruz Biotechnology Inc.) or anti-ERK1/2, antiphospho-AKT, and anti-phospho-NFATc1 antibodies (Cell Signaling Technology). Bands were visualized with enhanced chemiluminescence and quantified by densitometry (Image 1.41 software).

Primary cardiomyocyte isolations. Primary adult cardiomyocytes from $\operatorname{Sh} p 2^{\mathrm{Y} 279 \mathrm{C} /+}$, Shp $2^{\mathrm{Y} 279 \mathrm{C} / \mathrm{Y} 279 \mathrm{C}}$, and Tie2-Cre Shp $2^{\mathrm{Y} 279 \mathrm{C} /+}$ mice were isolated and cultured as previously described (66). Hearts were placed in a Langendorff apparatus, and adult cardiomyocytes were obtained by perfusion with $\mathrm{Ca}^{2+}$-free Tyrode buffer $\mathrm{pH} 7.4(135 \mathrm{mmol} / \mathrm{l} \mathrm{NaCl}$, $4 \mathrm{mmol} / 1 \mathrm{KCl}, 1 \mathrm{mmol} / 1 \mathrm{MgCl}_{2}, 0.33 \mathrm{mmol} / 1 \mathrm{NaH}_{2} \mathrm{PO}_{4}, 10 \mathrm{mmol} / \mathrm{l}$ HEPES), $10 \mathrm{mmol} / 1$ glucose, $10 \mathrm{mmol} / \mathrm{l}$ 2,3-butanedione monoxime (Sigma-Aldrich, catalog B0753), and $5 \mathrm{mmol} / \mathrm{l}$ taurine (Sigma-Aldrich, catalog T0625) for 3 to 5 minutes. Perfusion was continued for an additional 7 to 10 minutes with recirculating Tyrode buffer containing a mixture of collagenase D $(0.3 \mathrm{mg} / \mathrm{g}$ body weight; Roche), collagenase B (0.4 mg/g body weight; Roche), and proteinase XIV (0.05 mg/g body weight; Sigma-Aldrich). Ventricular tissue was then minced in Tyrode solution containing $2 \%$ bovine serum albumin (SigmaAldrich), incubated for 15 minutes at $37^{\circ} \mathrm{C}$, and filtered through a $250-\mu \mathrm{m}$ nylon mesh. The cell suspension was centrifuged at $420 \mathrm{~g}$ for 2 minutes and then gradually subjected to Tyrode buffer containing increasing concentrations of calcium and decreasing concentrations of 2,3-butanedione monoxime (i.e., the final concentration was 1.2 $\mathrm{mmol} / \mathrm{l} \mathrm{CaCl}_{2}$ with no 2,3-butanedione monoxime). Typical yields were $1.5 \times 10^{6}$ to $2.5 \times 10^{6}$ cells per heart, with $70 \%$ to $80 \%$ of the cells retaining their rod-shaped morphology. Cells were plated and digitally photographed using a Keyence BZ-X710 for cross-sectional area measurements using ImageJ; 200 to 500 cells per genotype from $n=3$ mice/group were measured.

Immune complex PTP assay. The PTP assay was conducted as previously described (20) using paranitrophenyl phosphate (pNPP; SigmaAldrich) as substrate. Briefly, mouse embryonic fibroblasts (MEFs)

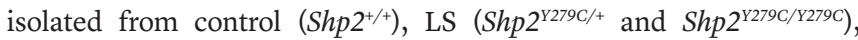
NS $\left(S h p 2^{D 61 G /+}\right)$, and $\operatorname{Sh} p 2$ null $\left(S h p 2^{-/-}\right)$embryos were cultured for 48 hours, serum-starved overnight, and then were either left unstimulated or were stimulated with IGF-1 $(10 \mathrm{nM})$ for 10 minutes. Cells were lysed in RIPA buffer (but without sodium orthovanadate). SHP2 was immunoprecipitated using anti-SHP2 polyclonal antibodies (Santa Cruz Biotechnology Inc.) coupled to protein A-Sepharose. Immune complexes were washed 3 times in RIPA buffer without sodium orthovanadate and once in wash buffer $(30 \mathrm{~mm}$ HEPES [pH 7.4], $120 \mathrm{~mm} \mathrm{NaCl}$ without pNPP). For each sample, PTP assays were performed in triplicate at $37^{\circ} \mathrm{C}$ in $50 \mu \mathrm{l}$ assay buffer $(30 \mathrm{~mm}$ HEPES [pH 7.4], $120 \mathrm{~mm} \mathrm{NaCl}, 5 \mathrm{~mm}$ dithiothreitol, $10 \mathrm{~mm}$ pNPP) containing $50 \mu$ lof the SHP2 beads. Reactions were terminated with $0.2 \mathrm{~N} \mathrm{NaOH}$, and phosphate release was determined by measuring $\mathrm{A}_{410}$. After the assays, immune complexes were recovered by cen- trifugation, boiled in $2 \times$ SDS-sample buffer, resolved by SDS-PAGE, and immunoblotted with polyclonal SHP2 antibodies (Santa Cruz Biotechnology Inc.) to ensure that equal amounts of SHP2 had been tested for phosphatase activity.

Statistics. All data are expressed as mean \pm SEM. Statistical significance was determined using 2-tailed Student's $t$ test or 1-way ANOVA, as appropriate. If ANOVA was significant, individual differences were evaluated using Bonferroni's post-test. For all studies, values of $P<0.05$ were considered statistically significant.

Study approval. All procedures were performed in accordance with the NIH Guide for the Care and Use of Laboratory Animals (National Academies Press. 2011.) and approved by the Institutional Animal Care and Use Committee at Beth Israel Deaconess Medical Center.

\section{Author contributions}

JL performed the majority of the experiments and analyzed the data. JRC and AR performed the experiments required for the revisions of the manuscript. KK conducted the cardiac analyses on adult LS mice. SMH, GCS, MEF, and LEM conducted the embryonic and myocardial tissue counts. FD and SD conducted the aortic regurgitation experiments and analyses. BW isolated the adult mouse cardiomyocytes. RB conducted the histology and analysis. KHL codirected parts of the study, analyzed data, and edited the paper. MIK directed the study, analyzed the data, and wrote the paper with input from all the authors.

\section{Acknowledgments}

We would like to thank Fabrice Jaffre from the Kontaridis laboratory for reading the manuscript. We would also like to thank Boding Zhang and Elizabeth G. Favre at the Medical University of South Carolina for their technical contribution to this manuscript and Robert J. Schwartz at the University of Houston and William Pu at Children's Hospital Boston for providing us with the Nkx2.5-Cre and cTnT-Cre mouse models, respectively. We would also like to thank Benjamin Neel for providing us the $\operatorname{Sh} p 2^{\mathrm{D} 61 \mathrm{G} /+}$ mice and the $\operatorname{Sh} p 2^{-/-}$MEFs. In addition, we would like to acknowledge and thank the funding sources for this project: NIH grants R01-HL102368, R01-HL114775, and RO1-HL122238 and a Children's Cardiomyopathy Foundation grant to M.I. Kontaridis, an American Heart Association Beginning Grant-in-Aid (12060120) and a South Carolina Translational Research seed grant to K. Lee, an American Heart Association postdoctoral fellowship to J. Lauriol, T32HL073734 to J.R. Cabrera and F. Damilano, and Summer Undergraduate Research grant 4R25HL092611-09 to G. Segarra and L.E. Miller. This work was also supported in part by the Beth Israel Deaconess Medical Center Division of Cardiology, the NIH/ NCATS UL1 TR001450 South Carolina Translational Research Institute (SCTR), South Carolina COBRE for Developmentally Based Cardiovascular Disease P2O 016434, and the Darby Children's Research Institute.

Address correspondence to: Maria Irene Kontaridis, Beth Israel Deaconess Medical Center, Department of Medicine, Division of Cardiology, Center for Life Sciences, Room 908, 3 Blackfan Circle, Boston, Massachusetts 02115, USA. Phone: 617.735.4248; E-mail: mkontari@bidmc.harvard.edu. 
1. Weismann CG, Gelb BD. The genetics of congenital heart disease: a review of recent developments. Curr Opin Cardiol. 2007;22(3):200-206.

2. Soor GS, et al. Hypertrophic cardiomyopathy: current understanding and treatment objectives. JClin Pathol. 2009;62(3):226-235.

3. Buckingham M, Meilhac S, Zaffran S. Building the mammalian heart from two sources of myocardial cells. Genetics. 2005;6(11):826-835.

4. Snider P, Olaopa M, Firulli AB, Conway SJ. Cardiovascular development and the colonizing cardiac neural crest lineage. ScientificWorldJournal. 2007;7:1090-1113.

5. Armstrong EJ, Bischoff J. Heart valve development: endothelial cell signaling and differentiation. Circ Res. 2004;95(5):459-470.

6. Zhang W, Chen H, Qu X, Chang CP, Shou W. Molecular mechanism of ventricular trabeculation/compaction and the pathogenesis of the left ventricular noncompaction cardiomyopathy (LVNC). Am J Med Genet C Semin Med Genet. 2013;163C(3):144-156.

7. Rentschler S, Jain R, Epstein JA. Tissue-tissue interactions during morphogenesis of the outflow tract. Pediatr Cardiol. 2010;31(3):408-413.

8. Wang Y, et al. Endocardial to myocardial notchwnt-bmp axis regulates early heart valve development. PLoS One. 2013;8(4):e60244.

9. Zhang $\mathrm{Y}$, et al. Foxp1 coordinates cardiomyocyte proliferation through both cell-autonomous and nonautonomous mechanisms. Genes Dev. 2010;24(16):1746-1757.

10. Greenway SC, et al. De novo copy number variants identify new genes and loci in isolated sporadic tetralogy of Fallot. Nat Genet. 2009;41(8):931-935.

11. Bauer RC, et al. Jagged1 (JAG1) mutations in patients with tetralogy of Fallot or pulmonic stenosis. Hum Mutat. 2010;31(5):594-601.

12. Wang B, et al. Foxp1 regulates cardiac outflow tract, endocardial cushion morphogenesis and myocyte proliferation and maturation. Development. 2004;131(18):4477-4487.

13. Tartaglia M, et al. Mutations in PTPN11, encoding the protein tyrosine phosphatase SHP-2, cause Noonan syndrome. Nat Genet. 2001;29(4):465-468.

14. Tartaglia M, Gelb BD. Noonan syndrome and related disorders: genetics and pathogenesis. Annu Rev Genomics Hum Genet. 2005;6:45-68.

15. Limongelli $\mathrm{G}$, et al. Prevalence and clinical significance of cardiovascular abnormalities in patients with the LEOPARD syndrome. Am J Cardiol. 2007;100(4):736-741.

16. Sarkozy A, et al. Clinical and molecular analysis of 30 patients with multiple lentigines LEOPARD syndrome. JMed Genet. 2004;41(5):e68.

17. Tartaglia M, Gelb BD, Zenker M. Noonan syndrome and clinically related disorders. Best Pract Res Clin Endocrinol Metab. 2011;25(1):161-179.

18. Yu ZH, et al. Structural and mechanistic insights into LEOPARD syndrome-associated SHP2 mutations. J Biol Chem. 2013;288(15):10472-10482.

19. Qiu W, et al. Structural insights into Noonan/ LEOPARD syndrome-related mutants of proteintyrosine phosphatase SHP2 (PTPN11). BMC Struct Biol. 2014;14:10.

20. Kontaridis MI, Swanson KD, David FS, Barford D,
Neel BG. PTPN11 (Shp2) mutations in LEOPARD syndrome have dominant negative, not activating, effects. J Biol Chem. 2006;281(10):6785-6792.

21. Fragale A, Tartaglia M, Wu J, Gelb BD. Noonan syndrome-associated SHP2/PTPN11 mutants cause EGF-dependent prolonged GAB1 binding and sustained ERK2/MAPK1 activation. Hum Mutat. 2004;23(3):267-277.

22. Araki T, et al. Mouse model of Noonan syndrome reveals cell type- and gene dosagedependent effects of Ptpn11 mutation. Nat Med. 2004;10(8):849-857.

23. Keilhack H, David FS, McGregor M, Cantley LC, Neel BG. Diverse biochemical properties of Shp2 mutants. J Biol Chem. 2005;280(35):30984-30993.

24. Niihori T, et al. Functional analysis of PTPN11/ SHP-2 mutants identified in Noonan syndrome and childhood leukemia. J Hum Genet. 2005;50(4):192-202.

25. Tartaglia M, et al. Diversity and functional consequences of germline and somatic PTPN11 mutations in human disease. Am J Hum Genet. 2006;78(2):279-290.

26. Schubbert S, et al. Functional analysis of leukemiaassociated PTPN11 mutations in primary hematopoietic cells. Blood. 2005;106(1):311-317.

27. Hanna N, et al. Reduced phosphatase activity of SHP-2 in LEOPARD syndrome: consequences for PI3K binding on Gab1. FEBS Lett. 2006;580(10):2477-2482.

28. Marin TM, et al. Rapamycin reverses hypertrophic cardiomyopathy in a mouse model of LEOPARD syndrome-associated PTPN11 mutation. JClin Invest. 2011;121(3):1026-1043.

29. Schramm C, Fine DM, Edwards MA, Reeb AN, Krenz M. The PTPN11 loss-of-function mutation Q510E-Shp2 causes hypertrophic cardiomyopathy by dysregulating mTOR signaling. Am J Physiol Heart Circ Physiol. 2012;302(1):H231-H243.

30. Tajan $\mathrm{M}$, et al. LEOPARD syndrome-associated SHP2 mutation confers leanness and protection from diet-induced obesity. Proc Natl Acad Sci U S A. 2014;111(42):E4494-E4503.

31. Neel BG, Gu H, Pao L. The 'Shp'ing news: SH2 domain-containing tyrosine phosphatases in cell signaling. Trends Biochem Sci. 2003;28(6):284-293.

32. Wu CJ, O'Rourke DM, Feng GS, Johnson GR, Wang Q, Greene MI. The tyrosine phosphatase SHP-2 is required for mediating phosphatidylinositol 3-kinase/Akt activation by growth factors. Oncogene. 2001;20(42):6018-6025.

33. Zhang SQ, et al. Receptor-specific regulation of phosphatidylinositol 3'-kinase activation by the protein tyrosine phosphatase Shp2. Mol Cell Biol. 2002;22(12):4062-4072.

34. Matsuo K, et al. Altered glucose homeostasis in mice with liver-specific deletion of Src homology phosphatase 2.J Biol Chem. 2010;285(51):39750-39758

35. Edouard T, et al. Functional effects of PTPN11 (SHP2) mutations causing LEOPARD syndrome on epidermal growth factor-induced phosphoinositide 3-kinase/AKT/glycogen synthase kinase $3 \beta$ signaling. Mol Cell Biol. 2010;30(10):2498-2507.

36. Yang W, et al. An Shp2/SFK/Ras/Erk signaling pathway controls trophoblast stem cell survival.
Dev Cell. 2006;10(3):317-327.

37. Jiang X, Rowitch DH, Soriano P, McMahon AP, Sucov HM. Fate of the mammalian cardiac neural crest. Development. 2000;127(8):1607-1616.

38. Kisanuki YY, Hammer RE, Miyazaki J, Williams SC, Richardson JA, Yanagisawa M. Tie2-Cre transgenic mice: a new model for endothelial cell-lineage analysis in vivo. Dev Biol. 2001;230(2):230-242.

39. Moses KA, DeMayo F, Braun RM, Reecy JL, Schwartz RJ. Embryonic expression of an Nkx25/Cre gene using ROSA26 reporter mice. Genesis. 2001;31(4):176-180.

40. Jiao K, et al. An essential role of Bmp4 in the atrioventricular septation of the mouse heart. Genes Dev. 2003;17(19):2362-2367.

41. Ma Q, Zhou B, Pu WT. Reassessment of Isl1 and Nkx2-5 cardiac fate maps using a Gata4based reporter of Cre activity. Dev Biol. 2008;323(1):98-104.

42. Grego-Bessa J, et al. Notch signaling is essential for ventricular chamber development. Dev Cell. 2007;12(3):415-429.

43. Lu SY, et al. FGF-16 is required for embryonic heart development. Biochem Biophys Res Commun. 2008;373(2):270-274.

44. Moon JB, et al. Akt induces osteoclast differentiation through regulating the GSK3beta/NFATc1 signaling cascade. JImmunol. 2012;188(1):163-169.

45. Bai S, Kerppola TK. Opposing roles of FoxP1 and Nfat3 in transcriptional control of cardiomyocyte hypertrophy. Mol Cell Biol.2011;31(14):3068-3080.

46. Kathiriya IS, Srivastava D. Left-right asymmetry and cardiac looping: implications for cardiac development and congenital heart disease. $\mathrm{Am} J$ Med Genet. 2000;97(4):271-279.

47. Bonetti M, Paardekooper Overman J, Tessadori F, Noel E, Bakkers J, den Hertog J. Noonan and LEOPARD syndrome Shp2 variants induce heart displacement defects in zebrafish. Development. 2014;141(9):1961-1970.

48. Langdon Y, Tandon P, Paden E, Duddy J, Taylor JM, Conlon FL. SHP-2 acts via ROCK to regulate the cardiac actin cytoskeleton. Development. 2012;139(5):948-957.

49. Nakamura T, Gulick J, Colbert MC, Robbins J. Protein tyrosine phosphatase activity in the neural crest is essential for normal heart and skull development. Proc Natl Acad Sci U S A. 2009;106(27):11270-11275.

50. Araki T, Chan G, Newbigging S, Morikawa L, Bronson RT, Neel BG. Noonan syndrome cardiac defects are caused by PTPN11 acting in endocardium to enhance endocardial-mesenchymal transformation. Proc Natl Acad Sci U S A. 2009;106(12):4736-4741

51. Chen B, et al. Mice mutant for Egfr and Shp2 have defective cardiac semilunar valvulogenesis. Nat Genet. 2000;24(3):296-299.

52. Person AD, Klewer SE, Runyan RB. Cell biology of cardiac cushion development. Int Rev Cytol. 2005;243:287-335.

53. Krenz M, Gulick J, Osinska HE, Colbert MC, Molkentin JD, Robbins J. Role of ERK1/2 signaling in congenital valve malformations in Noonan syndrome. Proc Natl Acad Sci U S A. 2008;105(48):18930-18935.

54. Mandinova A, et al. The FoxO3a gene is a key 
negative target of canonical Notch signalling in the keratinocyte UVB response. EMBO J. 2008;27(8):1243-1254.

55. Yugawa T, Handa K, Narisawa-Saito M, Ohno S, Fujita M, Kiyono T. Regulation of Notch1 gene expression by $\mathrm{p} 53$ in epithelial cells. Mol Cell Biol. 2007;27(10):3732-3742.

56. Ogawara Y, et al. Akt enhances Mdm2-mediated ubiquitination and degradation of p53. J Biol Chem. 2002;277(24):21843-21850.

57. de la Pompa JL, Epstein JA. Coordinating tissue interactions: Notch signaling in cardiac development and disease. Dev Cell. 2012;22(2):244-254.

58. van Boxtel R, et al. FOXP1 acts through a negative feedback loop to suppress FOXO-induced apoptosis. Cell Death Differ. 2013;20(9):1219-1229.
59. Ishida H, et al. LEOPARD-type SHP2 mutant Gln510Glu attenuates cardiomyocyte differentiation and promotes cardiac hypertrophy via dysregulation of Akt/GSK-3 $\beta / \beta$-catenin signaling. Am J Physiol Heart Circ Physiol. 2011;301(4):H1531-H1539.

60. Molkentin JD, et al. A calcineurin-dependent transcriptional pathway for cardiac hypertrophy. Cell. 1998;93(2):215-228.

61. Wilkins BJ, et al. Targeted disruption of NFATc3, but not NFATc4, reveals an intrinsic defect in calcineurin-mediated cardiac hypertrophic growth. Mol Cell Biol. 2002;22(21):7603-7613.

62. Xu M, et al. The endothelium-dependent effect of RTEF-1 in pressure overload cardiac hypertrophy: role of VEGF-B. Cardiovasc Res.
2011;90(2):325-334.

63. Nanba D, et al. Loss of HB-EGF in smooth muscle or endothelial cell lineages causes heart malformation. Biochem Biophys Res Commun. 2006;350(2):315-321.

64. Wilson CL, et al. Endothelial deletion of ADAM17 in mice results in defective remodeling of the semilunar valves and cardiac dysfunction in adults. Mech Dev. 2013;130(4-5):272-289.

65. Odelin G, et al. Loss of Krox20 results in aortic valve regurgitation and impaired transcriptional activation of fibrillar collagen genes. Cardiovasc Res. 2014;104(3):443-455.

66. Zhu X, et al. Three-dimensional structures of acidic and basic fibroblast growth factors. Science. 1991;251(4989):89-93. 